\title{
Profil alimentaire des ménages de la région de Kayes en février 2018
}

\author{
Household food profile of the Kayes region in February 2018
}

Sogoba A-k $k^{1}$, Ag lknane $A^{2}$, Dramé $M^{1}$, Ouologuèm $T^{1}$

\begin{abstract}
${ }^{1}$ Médecin Nutritionniste, Faculté de Médecine et d'Odontostomatologie, Université des Sciences, des Techniques et Technologies de Bamako (Mali). 2 Professeur en Santé Publique-nutrition, Faculté de Pharmacie, USTTB, Bamako (Mali)
\end{abstract}

\section{Résumé :}

La présente étude visait à évaluer les caractéristiques sociodémographiques et socioéconomiques des ménages associés au profil alimentaire de la région de Kayes en février 2018.

La méthode utilisée est une analyse secondaire des données de Kayes de l'enquête nationale de la sécurité alimentaire et nutritionnelle à travers une régression logistique multinomiale.

Les résultats révèlent que $33,8 \%$ des ménages avaient un score de consommation alimentaire pauvre.

Le score de consommation alimentaire était statistiquement associé au sexe, l'âge, la situation matrimoniale du chef de ménage, au statut de résidence et le quintile de richesse, la taille du ménage.

L'analyse de la diversité alimentaire montrait que 39,3\% des ménages avaient un score de diversité alimentaire élevé. Seul le statut était la seule variable qui n'avait pas de lien statistique avec le SDA.

Le score de consommation alimentaire, méthode permettant de connaitre la qualité du régime alimentaire, était associé à tous les caractéristiques socio démographiques du ménage.

Mots clés : Profil, alimentaire, ménages, Kayes

\section{Abstract:}

This study aimed to assess the sociodemographic and socioeconomic characteristics of households associated with food insecurity in the Kayes region in February 2018. The method used is a secondary analysis of Kayes data from the National Food and Nutrition Security Survey through a multinomial logistic regression.

The results reveal that $33,8 \%$ of households had a poor food consumption score.

The food consumption score was statistically associated with sex, age, marital status of head of household, residence status and wealth quintile, household size.

The food consumption score, a method of knowing the quality of the diet, was associated with all the sociodemographic characteristics of the household.

Key words: Household, food, profile, Kayes

\section{Introduction:}

Selon le Sommet mondial de l'alimentation (SMA) de 1996, la sécurité alimentaire existe quand toutes les personnes, à tout moment, ont un accès physique, social et économique à une nourriture suffisante, sûre et nutritive qui répond à leurs besoins diététiques et leurs préférences alimentaires, pour une vie saine et active [1].
L'Afrique est le continent le plus touché et l'insécurité est majoritairement concentrée en Afrique subsaharienne [2]. Le Mali est un pays sahélien, enclavé, et structurellement vulnérable à l'insécurité alimentaire et à la malnutrition.

La mise en œuvre de diverses interventions dans le cadre du Plan National de Réponse du Gouvernement en collaboration avec les agences humanitaires principalement dans le nord du pays a contribué à une stabilisation voire une amélioration de la sécurité alimentaire selon les résultats de l'ENSAN réalisée en septembre 2016 par le SAP, le PAM et leurs partenaires.. Selon les résultats de SMART 2017, la région de Kayes présente la prévalence la plus élevée de malnutrition aiguë globale et chronique avec [5].

Malgré les efforts consentis par le gouvernement du Mali pour lutter contre l'insécurité alimentaire, la situation reste précaire à Kayes d'où l'intérêt de cette étude.

\section{Matériels et méthodes :}

Une analyse secondaire des données d'une étude transversale descriptives par sondage stratifié en grappe à deux degrés reposant sur les données de l'Enquête Nationale sur la Sécurité Alimentaire et Nutritionnelle (ENSAN) de Février 2018 du Mali pour la région de Kayes. L'analyse des données a été effectuée à l'aide du logiciel SPSS version 21.0. Des tests statistiques de khicarré de Pearson et de régression logistique ont été réalisés avec un degré de signification de $5 \%$.

\section{Résultats :}

Les chefs de ménages (CM) étaient en majorité des hommes $(94,6 \%$ ), mariés $(93,9 \%)$ dont $50,1 \%$ de monogames et $44,7 \%$ de polygames. Près de $60,4 \%$ des hommes chefs de ménages étaient sans instruction. L'âge moyen des chefs de ménages était de $52+$ ou 13,97 ans. Les ménages appartenant au quintile des plus pauvres représentaient 21,5 contre $47,9 \%$ de ceux du quintile des plus riches.

Le score de consommation alimentaire (SCA) des ménages était pauvre dans $50,4 \%$ et un score de diversité alimentaire (SDA) de 39,3\%. Le nombre moyen de groupes d'aliments consommés la veille était inférieur à 4 .

Après ajustement, toutes les caractéristiques étaient statistiquement associées à la consommation alimentaire $(p=0,0001)$. Cependant seules les pauvres avaient plus de risque de ne pas avoir une alimentation adéquate. Par contre les résidents $(\mathrm{ORa}=0,183)$, les instruits $(\mathrm{ORa}=$ 
$0,470)$, les non-mariés $(0,469)$, les chefs de ménage de moins de 50 ans (ORa= 739), les ménages de moins de 17 personnes $(0 \mathrm{Ra}=0,709)$ avaient plus de chance d'avoir une alimentation adéquate. Egalement, toutes les caractéristiques sauf la résidence étaient statistiquement associées à la diversité alimentaire $(p=0,0001)$. Les nonmariés $(\mathrm{ORa}=1,462)$, les moins de 50 ans $(\mathrm{ORa}=1,52)$ avaient plus de risque de ne pas avoir une alimentation diversifiée. Par contre les ménages de moins de 17 personnes $(\mathrm{ORa}=0,874)$, les instruits $(\mathrm{ORa}=0,587)$, les riches avaient plus de chance d'avoir une alimentation diversifiée.

\section{Discussion}

L'âge moyen du chef de ménage est de 52 ans. Ce résultat est semblable à celui de [ENSAN Mali, Février 2017] [5] qui trouve que la moyenne d'âge des chefs de ménage était de 52 ans.

La tranche d'âge ayant une alimentation adéquate et diversifiée est celle des plus de 50 ans. Cette situation s'expliquerait par le fait que cette classe d'âge est plus entretenue sur le plan alimentation que les moins de 50 ans.

II ressort de l'enquête que les ménages dirigés par des hommes étaient plus nombreux à Kayes $(94,6 \%)$. Cela semble normal étant donné que le code de famille malien reconnaît que l'époux (l'homme) est le chef de ménage dans le cas d'un couple marié.

Nos résultats sont comparables à ceux de l'enquête [ENSAN, Février 2017] [5] qui trouve 92,8\% des ménages sont dirigés au niveau national et différent de [ENSAN Sénégal, 2016] [14] qui trouve que 70\% des ménages étaient dirigés par les femmes.

Les chefs de ménage étaient monogames à $50,1 \%$. Nos résultats sont comparables celui de [ENSAN Mali, Septembre 2016] [4] qui trouve $56,8 \%$ de régime monogamique.

Les non mariés avaient plus de chance d'avoir une alimentation adéquate et diversifiée par rapport aux mariés. Ce qui pourrait s'expliquer par le fait que les non mariés peuvent bénéficier du soutien et ou d'aides d'autres personnes.

Les chefs de ménages étaient à $60,4 \%$ sans instruction. Ce résultat est inférieur à celui de l'enquête [ENSAN Mali, Février 2017] [5] qui trouve que le taux d'analphabétisme était plus élevé à Kayes avec 71,2\%. Les non instruits avaient plus de chance de ne pas avoir une alimentation diversifiée que les instruits. Cette relation pourrait être attribuée par le niveau de connaissances de façon générale et en particulier par la compréhension et la connaissance de l'importance d'une bonne alimentation sur la santé.

II n'y a pas de lien entre la résidence et la diversification alimentaire par contre, les non-résidents ont plus de chance d'avoir une alimentation adéquate que les résidents. Ce résultat est semblable à celui de $\mathrm{M}$. DRAME à Bamako en 2018[18] qui trouve que les non- résidents à Bamako ont plus de chance d'avoir une alimentation adéquate par rapport aux résidents.

Les pauvres ont plus de risque de ne pas avoir une alimentation adéquate que les riches par contre les pauvres ont plus de chance d'avoir une alimentation diversifiée que les riches.

Ce résultat pourrait être attribué par le fait que les pauvres se soucient en premier lieu à se nourrir, consacrent la majeur partie de leurs ressources à l'alimentation, bénéficient souvent d'aides d'autres personnes. Ce résultat est semblable à celui de [E.PAUZE Haïti, 2015] [17] qui trouve que la diversité alimentaire est liée au statut socioéconomique.

\section{Conclusion}

L'âge, le sexe, le quintile de richesse, la situation matrimoniale, la résidence du chef de ménage sont statistiquement associés au score de consommation alimentaire.

L'étude nous montre que la diversité alimentaire est liée aux caractéristiques sociodémographiques économiques des ménages. Seule la résidence n'a pas de lien statistique avec la diversité alimentaire.

\section{Références}

1- FAO, FIDA et PAM. (2014). L'État de l'insécurité dans le monde 2014. Créer un environnement plus propice à la sécurité alimentaire et à la nutrition. Rome: FAO, 57 pages. http://www.fao.org/3/a-i4030f.pdf. (Consulté le 10/11/2018)

2-FAO, FIDA et PAM. 2012. L'État de l'insécurité alimentaire dans le monde 2012. La croissance économique est nécessaire mais elle n'est pas suffisante pour accélérer la réduction de la faim et de la malnutrition. Rome, http://www.fao.org/docrep/016/i2845f/i2845f00.pdf (consulté le 10/11/21018)

3-MSHP, INSTAT, UNICEF, PAM, OMS, FAO. Enquête Nationale Nutritionnelle et de Mortalité Rétrospective suivant la méthodologie SMART, Mali, août 2017, http://instat

mali.org/contenu/eq/rafsmart17_eq.pdf (consulté le 29/12/2018)

4- INSTAT. Enquête Nationale Nutritionnelle Anthropométrique et mortalité Rétrospective Mali. 2011. pp :(29-30), 80p.

SAP, WFP FAO FEWS NET et CLUSTER SECURITE ALIMENTAIRE. Enquête Nationale sur la sécurité alimentaire et nutritionnelle, Septembre 2016 (ENSAN MALI)- (consulté le 12/10/2018)

5-SAP, WFP FAO FEWS NET et CLUSTER SECURITE

ALIMENTAIRE. Enquête Nationale sur la sécurité alimentaire et nutritionnelle, février 2017 (ENSAN MALI)(consulté le 12/11/2018)

6- Secrétariat Exécutif du Conseil de Sécurité Alimentaire du Sénégal, PAM et World Vision : Enquête

Nationale sur la sécurité alimentaire et nutritionnelle, 
Septembre 2016 (ENSAN Sénégal). https://documents.wfp.org/stellent/groups/public/docu ments/ena/wfp292119.pdf?iframe (consulté le 22/11/2018)

7- FAO. Sécurité alimentaire: Sommet mondial de l'alimentation Rome, 16 - 18 novembre 2009, http://www.fao.org/fileadmin/templates/wsfs/Summit/ Docs/Declaration/K6050REV10F.pdf (consulté le 16/11/2018)

8-Popkin BM. Urbanization, lifestyle changes and the nutrition transition. World Development 1999; University of North Carolina, Chapel Hill, USA 27(11): 1905-16.

https://pdfs.semanticscholar.org/b283/26f9187cb726 028e296e224590330580f71d.pdf (consulté le $14 / 11 / 2018)$

9-FAO (1983). Comité de la sécurité alimentaire mondiale (CFS 83/4): Rapport du Directeur général sur la sécurité alimentaire mondiale: Une réévaluation des concepts et approches. Rome. http://www.fao.org/docrep/meeting/026/MD776F.pdf (consulté le 16/11/2018)

10- Geographie de la région de Kayes

https://fr.wikiperdia.org/wikiR\%//C3\%A9gion de Kayes 11- -FAO. Rapport dynamique temporelle de l'insécurité alimentaire (Clay, 2002)

$\mathrm{ftp}$ //fttp.fao.org/es/ESA/policybriefs/pb_02_fr.pdf (consulté le 16/11/2018)

12- FAO.Sous-alimentation,

http://sousalimentation.canalblog.com/ (consulté le 18/11/2018)

13- P. HORDE. Ration alimentaire, février 2014

http://sante-medecine.journaldesfemmes.com/faq/22391 . ration-alimentaire-definition (consulté le 19/11/2018)

14-- PAM. Score de consommation et de diversité alimentaire, Dakar Atelier Régional de Formation: 1012 Juin 2014

http://www.fao.org/fileadmin/user_upload/foodsecurity capacitybuilding/docs/Nutrition/SahelWorkshop/2.2.W FP_Int\%C3\%A9grationIndicateurse tut.pdf (consulté le 19/11/2018)

15-PAM. Enquête approfondie de la sécurité alimentaire et de la vulnérabilité des ménages ruraux en Guinée Bissau, Mars 2011

https://www.wfp.org/sites/default/files/Guinee-BissauFood $\% 20$ Security $\% 20$ assessment $\% 20$ Executive $\% 20$ Brief_Final_0.pdf (consulté le 22/11/2018)

16-Union Européenne, SOS SAHEL. Enquête de base : Situation de Référence du PRIAN - Programme Key dans les Régions de Mopti, Tombouctou et Kidal SocioEconomique et nutrition, juillet 2017

https://fscluster.org/sites/default/files/documents/rapport prian_25092017.pdf (consulté le 23/11/2018)

17- E. Pauzé. La qualité de l'alimentation et l'accès alimentaire des ménages vulnérables habitant dans une zone d'intervention d'agriculture de santé publique en Haïti, 2015.

https://ruor.uottawa.ca/bitstream/10393/32432/3/Pau
Z\%C3\%A9_Elise_2015_thesis.pdf(consulté le 24/11/2018)

18-M. DRAME. Caractéristiques sociodémographiques associés à la consommation alimentaire des ménages du district de Bamako, septembre 2017. (Consulté le 24/11/2018)

19- PAM. Système de Suivi et surveillance de la Sécurité Alimentaire en Djibouti, octobre 2016.

https://docs.wfp.org/api/documents/WFP0000069359/download/?iframe (consulté le 24/11/2018)

20- INSTAT. Enquête modulaire et permanente auprès des ménages (EMOP) mai 2016,

http://www.instat-

mali.org/contenu/eq/ranuel15_eq.pdf (consulté le 25/11/2018)

21-FAO. Évolution des concepts de politique en matière de sécurité alimentaire, (Clay, 2002; Heidhues et al, 2004)

http://ftp.fao.org/es/ESA/policybriefs/pb_02_fr.pdf (consulté le 15/11/2018)

22-FAO. Evolution des concepts de politique en matière de sécurité alimentaire, (Clay, 2002 ;Heidhues et al, 2004)

http://ftp.fao.org/es/ESA/policibriefs/pb_02_fr.pd (consulté le 15/11/2018) 
Tableau I : Caractéristiques sociodémographiques et économiques des ménages de la population en février 2016 dans la région de Kayes

\begin{tabular}{|c|c|c|c|c|c|c|}
\hline \multirow[b]{2}{*}{ Variables } & \multicolumn{3}{|c|}{ Consommation alimentaire adéquate } & \multicolumn{3}{|c|}{ Alimentation diversifiée } \\
\hline & $\mathrm{OR}_{\mathrm{a}}$ & $\mathrm{IC}_{95 \%}$ & P-value & $\mathrm{OR}_{\mathrm{a}}$ & $\mathrm{IC}_{95 \%}$ & P-value \\
\hline \multicolumn{7}{|l|}{ Milieu de résidence } \\
\hline Non Résident & 1 & & & 1 & & \\
\hline Résidence & 3,084 & $2,621-3,628$ & 0,0001 & l & l & l \\
\hline \multicolumn{7}{|l|}{ Niveau d'instruction } \\
\hline Non instruit & 1 & & & & & \\
\hline Instruit & 1,834 & $1,788-1,881$ & 0,0001 & 1,734 & $1,629-1,846$ & 0,0001 \\
\hline \multicolumn{7}{|l|}{ Sexe } \\
\hline Masculin & 1 & & & & & \\
\hline Féminin & 1,951 & $1,808-2,112$ & 0,0001 & 0,579 & $0,509-0,659$ & 0,0001 \\
\hline \multicolumn{7}{|l|}{ Tranche d'âge } \\
\hline $20-45$ ans & 1 & & & 1 & & \\
\hline 46 ans et plus & 1,448 & $1,413-1,484$ & 0,0001 & 0,618 & $0,584-0,655$ & 0,0001 \\
\hline \multicolumn{7}{|l|}{ Statut Matrimonial du CM } \\
\hline Mariés & 1 & & & 1 & & \\
\hline Non Mariés & 1,331 & $1,234-1,435$ & 0,0001 & 1,220 & $1,060-1,405$ & 0,0001 \\
\hline \multicolumn{7}{|l|}{ Taille du ménage } \\
\hline Moins de 17 personnes & 1 & & & 1 & & \\
\hline Plus de 17 personnes & 2,002 & $1,591-2,055$ & 0,0001 & 1,140 & $1,073-1,211$ & 0,0001 \\
\hline \multicolumn{7}{|l|}{ Bien être économique } \\
\hline Pauvres-Moyens & 1 & & & 1 & & \\
\hline Riches & 0,143 & $0,138-1,148$ & 0,0001 & 1,343 & $1,250-1,442$ & 0,0001 \\
\hline
\end{tabular}

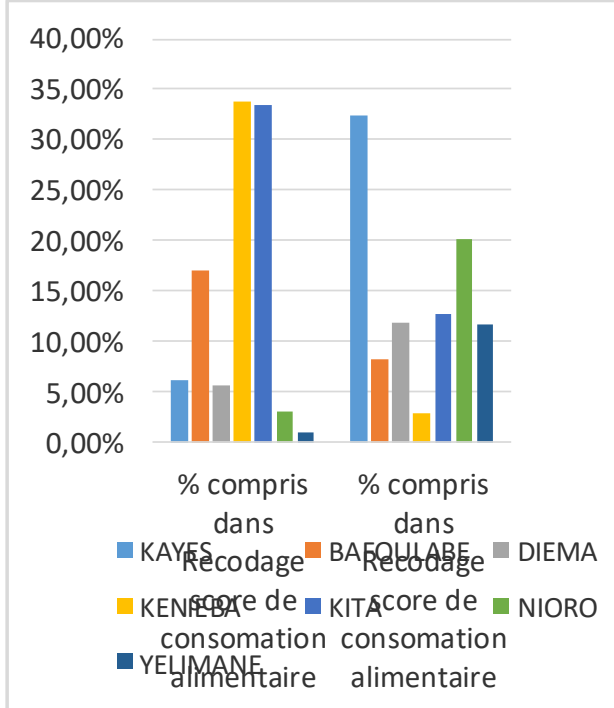

Figure 1 : Répartition de la consommation alimentaire des ménages de la région de Kayes en février2018

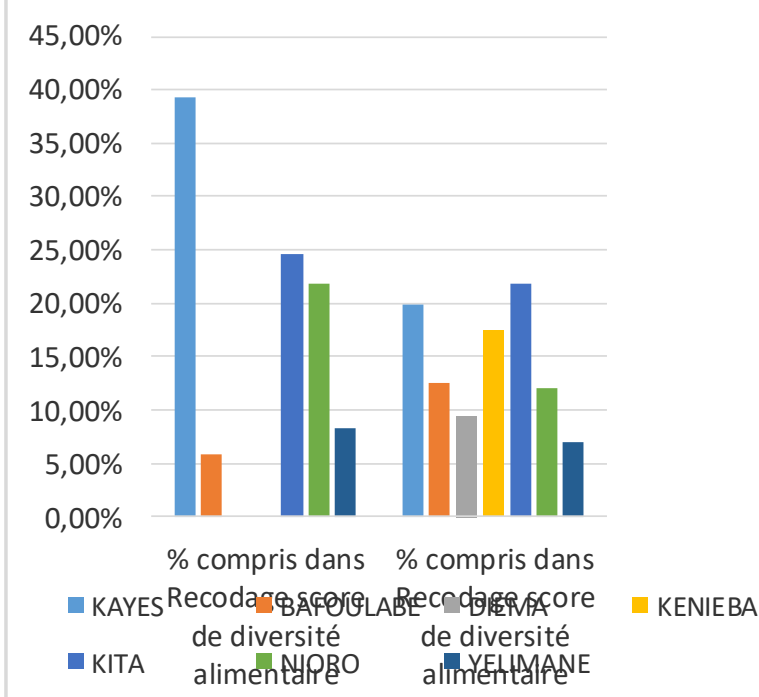

Figure 2 : Répartition de la diversité alimentaire des ménages de la région de Kayes en février 2018 
Le niveau de satisfaction des patients hospitalisés dans le district sanitaire de Koutiala au Mali en 2019

\title{
The level of satisfaction of hospitalized patients in the health district of Koutiala in Mali in 2019
}

\author{
Sanogo $B^{1,4^{*}}$, Diarra $B^{2,4}$, Sangho $H^{3,4} A g$ Iknane $A^{3,4}$
}

\author{
'Titulaire d'un Master II Santé publique, Option : Santé \\ Communautaire \\ ${ }^{2}$ Maître assistant en Santé Publique \\ ${ }^{3}$ Professeur titulaire de Santé Publique \\ ${ }^{4}$ Département d'Enseignement et de Recherche en Santé \\ Publique et spécialité, Faculté de Médecine et d'Odonto- \\ Stomatologie, Université des Sciences, des Techniques et des \\ Technologies de Bamako, Mali. \\ "Auteur Principal : Dr SANOGO Boubacar, Master II Santé \\ Publique, Option : Santé Communautaire, Département \\ d'Enseignement et de Recherche en Santé Publique et \\ spécialités (DERSP), Faculté de Médecine et \\ d'Odontostomatologie (FMOS) de Bamako, Mali. Tel : +223 76 \\ 3783 49/+22364 44 19 12; E-Mail : \\ drsanogoboubacar@gmail.com
}

\section{Résumé}

Introduction: Le respect des besoins et souhaits des patients est central dans tout système de santé. Présentée comme un paramètre d'évaluation des services publics, la satisfaction des usagers est considérée comme un indicateur de la qualité des soins. C'est dans cette optique et suite à de multiples plaintes de la part des usagers que nous avons jugé utile d'étudier la satisfaction des patients hospitalisés dans le district sanitaire de Koutiala. Méthodes: Une étude transversale mixte portant sur 83 patients a été mené de Juin à Juillet 2019. Le questionnaire comparis.ch adapté et traduit en langue locale (Bambara) pour accessibilité a été administré en face à face. Résultats: Avec un sexratio de 0,63 en faveur du sexe féminin, l'âge moyen était $39,3 \pm 19,5$ ans avec des extrêmes de 11-85 ans. Les non scolarisés représentaient $51,81 \%$. La tranche d'âge 18-34 ans représentaient $41 \%, 47 \%$ provenaient des communes urbaines de Koutiala. Le taux de satisfaction globale était $94 \%$. Les principaux éléments associés à une bonne satisfaction étaient: la confiance aux soignants $(p=0,002)$, le délai d'attente en cas de besoin $(p=0,04)$, la sécurité des soins $(p=0,017)$, l'intention de recommander $\left(p=10^{-3}\right)$, la qualité des prestations $(p=$ $\left.10^{-3}\right)$. Ceux incriminés dans l'insatisfaction étaient: l'exigence d'une caution $(p=0,003)$, la vente de médicament par les soignants. Conclusion: La satisfaction globale des patients hospitalisés était liée à une amélioration de leur état de santé, cependant les points d'insatisfactions énumérés constituent des thèmes privilégiés d'action.

Mots Clés: Satisfaction, patients, hospitalisés, Koutiala, Mali

\section{Abstract}

Introduction: Respect for the needs and wishes of patients is central to any health system. Presented as a parameter of evaluation of the public services, the satisfaction of the users is considered as an indicator of the quality of the care. It is in this perspective and following multiple complaints from users that we have found it useful to study the satisfaction of patients hospitalized in the health district of Koutiala. Methods: A mixed cross-sectional study involving 83 patients was conducted from June to July 2019. Comparis.ch adapted and translated into local language (Bambara) for accessibility was administered face-to-face. Results: With a sex ratio of 0.63 for females, the mean age was $39.3 \pm 19.5$ years with extremes of $11-85$ years. Out-ofschool children accounted for $51.81 \%$. The age group 18 34 years accounted for $41 \%, 47 \%$ came from the urbane communes of Koutiala. The overall satisfaction rate was $94 \%$. The main elements associated with good satisfaction were: confidence in caregivers $(p=0.002)$, waiting time when needed $(p=0.04)$, safety of care $(p=0.017)$, intention to recommend $\left(p=10^{-3}\right)$ the quality of services $\left(p=10^{-3}\right)$. Those incriminated in the dissatisfaction were: the requirement of a surety $(p=0.003)$, the sale of medicine by the caregivers. Conclusion: The overall satisfaction of hospitalized patients was correlated with an improvement in their state of health; however, the points of dissatisfaction enumerated are preferred themes of action.

Key words: Satisfaction, patients, hospitalized, Koutiala, Mali

\section{Introduction}

Présentée comme un paramètre d'évaluation des services publics, la satisfaction des usagers est considérée comme un indicateur de la qualité des soins $[1,2]$. En outre, elle revêt une importance plus globale dans la qualité des soins, car elle permet de valoriser la relation patient-soignant et d'instaurer un climat de confiance dans la sphère des soins. Elle est liée à l'adhésion thérapeutique, à la continuité des soins et à l'amélioration de l'état de santé [3,4].

Selon I'Organisation Mondiale de la Santé : " L'évaluation de la qualité des soins est une démarche qui permet de garantir à chaque patient des actes diagnostiques et thérapeutiques assurant le meilleur résultat en terme de santé, conformément à l'état actuel de la science médicale, au meilleur coût pour le meilleur résultat, au moindre risque iatrogène et pour sa plus grande satisfaction en termes de procédures, de résultats et de contacts humains à l'intérieur du système de soins » [5].

Le malade n'est plus un consultant ou un usager mais un client, au même titre que sa famille. Et ce client est devenu plus exigeant quant à la qualité des services. II considère la santé comme un droit, un service dû ; l'importance de ses cotisations sociales le pousse à avoir un œil critique [1]. 
La littérature sur les études de la satisfaction des patients hospitalisés montre que le concept de satisfaction est multidimensionnel et de nombreux facteurs (caractéristiques sociodémographiques, de santé, de structure de soins...) peuvent influencer cette mesure. Deux aspects sont classiquement identifiés sous le terme de satisfaction: l'aspect technique et la dimension humaine, relationnelle et de confort. Le patient juge de l'aspect technique du traitement d'une part, et d'autre part exprime son avis sur la façon dont il a été accueilli, le temps d'attente, l'information qu'il a ou non reçue, la disponibilité des agents de santé, la salubrité des locaux etc... $[1,6]$.

L'intérêt porté à la qualité des soins n'a cessé de croître ces dernières années. Dans bon nombre de pays, la majorité des établissements de santé la placent au cœur de leurs priorités [4].

L'idée que les usagers peuvent contribuer à l'amélioration de la qualité des services de santé par le biais de sondages a pris forme au cours des années 1970. C'est à ce moment qu'on a eu recours, d'abord aux États-Unis, aux premiers sondages de satisfaction des usagers [7]. En France, la mesure de la satisfaction des patients est une obligation réglementaire pour les établissements hospitaliers depuis l'ordonnance du 24 avril 1996 [8].

Au Mali, depuis les années 80 , les hôpitaux ont été confrontés à des problèmes de performance. Pour remédier à ces insuffisances, il y a eu la Réforme Hospitalière avec création de la Mission d'Appui à la Réforme Hospitalière (MARH) suivant le Décret $n^{\circ}$ 99105 / PM-RM du 12 mai 1999. Elle a fixé les objectifs suivants: placer le malade au cœur de l'hôpital et restructurer les hôpitaux (la gestion, la qualité des soins, le coût et l'accessibilité des soins. A cela s'ajoute la loi $n^{\circ}$ 02-050 / du 22 Juillet 2002 modifiée par la loi n¹8-050 portant loi hospitalière, la loi $n^{\circ} 02-049$ / du 22 Juillet 2002 modifiée par la loi n¹8-049 portant loi d'orientation sur la santé, l'Arrêté n08-2716 / MS-SG du 6 Octobre 2008 portant charte du malade [9-12].

Nonobstant tous ces faits, pourtant, une grande insatisfaction persiste, du point de vue des populations. D'ailleurs, le rapport d'étude d'évaluation de la mise en œuvre de la réforme hospitalière au Mali effectuée en 2010 par l'Agence Nationale d'Evaluation des hôpitaux (ANEH) repère des insuffisances dans sa mise en œuvre qui ont eu des conséquences parmi lesquelles : « la non satisfaction de la majorité des usagers » [9].

Suite à une pré-enquête à trois étapes au district sanitaire de Koutiala, les clients rencontrés ont évoqué un certain nombre de griefs comme motif de non satisfaction. Raison pour laquelle, après avoir échangé avec quelques agents, nous avons trouvé que ces derniers estimaient bien travailler, contrairement aux allégations de certains bénéficiaires.

Hormis les évaluations annuelles de l'ANEH, où la satisfaction des usagers est évaluée depuis 2006, il n'existe pas un système de suivi de la satisfaction des patients, ni un texte réglementaire imposant sa mesure au niveau des structures sanitaires au Mali.

En plus, nous n'avons pas trouvé de référence sur les enquêtes d'évaluation de la satisfaction des patients hospitalisés au Mali de façon générale, ni à Koutiala de façon particulière. Alors, nous avons trouvé utile de porter notre choix sur ce thème, à travers la présente étude dont l'objectif est d'étudier la satisfaction des patients hospitalisés au district sanitaire de Koutiala.

\section{Matériels et méthode}

\section{Présentation du cercle de Koutiala}

La fondation de Koutiala remonterait au 16ème et 17ème siècle. Koutiala aurait été fondé par les SANOGO, venus de Sanga, petit village situé à $5 \mathrm{~km}$ de la ville. Créé en 1903, le cercle de Koutiala, dans la région de Sikasso, est situé au Nord-Ouest de la 3ième région administrative du Mali. II compte 337 villages repartis entre une commune urbaine (Koutiala) et 35 communes rurales. Selon l'annuaire statistique produit par le système local d'information sanitaire de la région de Sikasso, le cercle de Koutiala avait une population estimée à 773966 habitants en 2018 avec un taux d'accroissement de 3,6\% soit une densité moyenne de 63 habitants/km2 (District sanitaire de Koutiala). Avec une superficie de 12.270 km2, le cercle est limité au nord et au nord-ouest par les cercles de Bla et San (Région de Ségou) au sud par le cercle de Sikasso et le Burkina Faso, à l'est par le cercle de Yorosso et à l'ouest par le cercle de Diola (Région de Koulikoro) [13-15].

\section{Dispositif d'offre des soins et services au district} sanitaire de Koutiala

Le district sanitaire organise non seulement des services médicaux mais aussi des services spéciaux. Dans le premier cas il y a : le service d'accueil des urgences, la consultation générale, l'hospitalisation, la maternité, la médecine interne et la chirurgie générale alors que dans le second il y a : la gynécologie obstétrique, la pédiatrie, la nutrition, l'odontostomatologie, la gastro-entérologie, la psychiatrie, l'ophtalmologie et l'urologie. A cela, il convient d'ajouter qu'ils y sont menés hebdomadairement des activités de santé communautaires (Consultations Prénatales, Programme Elargie de Vaccination, Planification Familiale) à l'occasion desquelles est organisée la communication pour le changement de comportement. II y a aussi deux pharmacies (nuit et jour) fonctionnelles. A cela s'ajoutent l'imagerie (radiographie et échographie), et le laboratoire d'analyse. Cependant, il convient de signaler qu'un partenariat existe entre le district sanitaire de Koutiala et I'ONG Médecins Sans Frontière France (MSFF) assurant le volet « pédiatrienutrition " au sein du district sanitaire. Pour ce qui est des infrastructures existantes, le district sanitaire de Koutiala dispose d'environ 17 blocs parmi lesquelles un bloc administratif, un bloc destiné au service d'accueil des urgences, un bloc destiné à la maternité, deux blocs d'hospitalisation de médecine générale (homme et dame), un bloc d'hospitalisation de la médecine interne, 
un bloc destiné au laboratoire d'analyse, un bloc opératoire, des blocs destinés au Programme Elargie de Vaccination, au Programme de Planification Familiale, à la pédiatrie-nutrition, à l'odontostomatologie, à l'ophtalmologie, à la chaine de froid et la morgue.

3. Type et période d'étude

II s'agissait d'une étude transversale, mixte (quantitative, qualitative) et descriptive dont la collecte s'est déroulé du 11 Juin au 11 Juillet 2019 soit un mois.

\section{Population d'étude}

La population d'étude était constituée de l'ensemble des usagers fréquentant le district sanitaire de Koutiala, tant ceux qui étaient hospitalisés que ceux qui fréquentaient le centre en ambulatoire.

Ont été inclus dans cette étude: tout patients hospitalisés dans le district sanitaire de Koutiala pendant au moins 24 heures au cours de la période d'enquête ; les parents (garde-malades) des patients de moins de 18 ans et des malades dont l'état de santé ne permettait pas de répondre à l'interview. Ont été exclus de l'enquête les patients qui ont utilisé le district sanitaire en ambulatoire ou qui ont refusé de participer à l'enquête.

L'échantillonnage concerna l'ensemble des patients hospitalisés dans les quatre (4) unités d'hospitalisation à savoir : la chirurgie générale (l'unité homme et l'unité dame), la maternité et la médecine interne. Le projet MSFF s'occupe du volet "Nutrition - Pédiatrie » offrant les soins et services gratuitement raison pour laquelle ils n'ont pas été inclus dans notre étude.

La taille de l'échantillon a été calculée avec l'outil STATCALC du logiciel Epi info Version 7.2.2.2. Avec $\mathrm{N}=$ nombre de patients hospitalisés en moyenne par mois en 2018 dans les 4 unités = $100 ; a=$ risque d'erreur $5 \% ; Z$ = écart réduit $=1,96 ; p=$ taux de satisfaction $73 \% ; \mathrm{Ce}$ taux a été choisi car d'une part nous n'avions pas de donner sur la satisfaction des patients hospitalisés dans le district sanitaire de Koutiala et d'autre part c'était le taux moyen de satisfaction trouver par l'Agence National d'Evaluation des Hôpitaux (ANEH) en 2018 dans son étude auprès des usagers des 13 établissements hospitaliers du Mali [16]. Cette méthode nous donna un effectif de 75 patients. Afin d'augmenter la puissance de notre étude, nous avons ajouté $10 \%$ de la taille de l'échantillon, ce qui revenait à 83 patients à enquêter.

\section{Outils de collecte}

La collecte des données a été faite à l'aide de questionnaires administrés en face à face. Si le patient avait été hospitalisé plus d'une fois durant la période de l'étude, il n'était interviewé qu'une seule fois. Le questionnaire comparis.ch adapté à notre contexte et traduit en langue locale (Bambara) pour être accessible à nos patients a été utilisé. Les questions étaient fermées, semi-ouvertes et à échelle pour certaines.

Les informations recueillies étaient : caractéristiques sociodémographiques, accompagnement social reçu, prestations offertes et organisation des soins et services, mode de facturation des actes, satisfaction globale.

6. Prétest
Un prétest a été organisé au niveau du district sanitaire de la commune $\mathrm{V}$ du district de Bamako. A cette occasion, nous avons interrogé 6 patients hospitalisés. Cet exercice nous avait permis d'apporter des amendements au questionnaire en fonction de la difficulté qu'il y avait à comprendre certaines questions de la part des répondants.

\section{Traitement et analyse des données}

La saisie et l'analyse des données ont été faites à travers le logiciel SPSS.25. Les résultats ont été présentés sous forme de tableaux en utilisant Microsoft Word 2019. Le test exact de Fisher a été utilisé pour analyser le lien entre la satisfaction globale des patients et les facteurs prédictifs car tous les tableaux croisés contenaient au moins un effectif théorique inférieur à 5. Pour un $p>0,05$ la différence est statistiquement non significative alors que pour un $p<0,05$ la différence est statistiquement significative.

\section{Considérations éthiques}

Cette recherche a été faite dans le strict respect de l'intégrité et la dignité des usagers du district sanitaire de Koutiala. Pour cela, nous avons expliqués le bien-fondé de la recherche, afin que les répondants soient éclairés et adhèrent volontairement et en connaissance de cause. Avant l'obtention du consentement oral, les participants ont été aussi rassurer quant au caractère purement scientifique de cette investigation, tout en leur expliquant que les résultats pourront aider les décideurs à améliorer la qualité des soins et services au sein du district sanitaire de Koutiala, pour le bien des usagers et du personnel et ainsi améliorer la santé de la communauté.

\section{Résultats}

\section{Caractéristiques sociodémographiques}

L'échantillon de patients était constitué de 32 hommes $(38,6 \%)$ et 51 femmes $(61,4 \%)$. Les catégories d'âges 18 - 34 ans et les 65 ans et plus étaient les plus représentées avec respectivement $41 \%$ et $21,7 \%$. L'âge moyen était de 39,3 \pm 19,5 avec des extrêmes de 11 à 85 ans. Parmi les répondants, les mariés étaient les plus représentés avec $84,3 \%$. Avec 43 répondants soit $51,8 \%$, les non scolarisés étaient les plus représentés. Près de la moitié des répondants provenaient des communes rurales de Koutiala soit $47 \%$. Avec respectivement 36,1\% et $30,1 \%$, les cultivateurs et les ménagères étaient les plus représentés (Tableau I).

\section{Accompagnement social reçu}

Parmi les répondants $94 \%$ estimaient avoir reçu un bon accueil de la part du personnel. Parmi les répondants enquêtés $92,8 \%$ avaient confiance aux soignants. "IIs soignent bien " et " Ils accueillent bien " ont été incriminé comme motifs de confiance au personnel par respectivement 62,3 et $35,1 \%$ des répondants. La presque totalité des enquêtés $(95 \%)$ estimaient qu'il y a collaboration entre ses proches (familiers) et les soignants. La presque totalité des répondants $(95,2 \%)$ estimaient être traité en toute dignité, $88,6 \%$ déclaraient avoir eu des réponses claires aux questions qu'ils avaient posées. Au district sanitaire de Koutiala 92,8\% 
des répondants mettaient moins de cinq minutes à attendre les soignants en cas de besoin et estimaient raisonnable ce délai d'attente, $94 \%$ avaient fortement l'intention de recommander le district sanitaire à un proche (Tableau II).

Prestations offertes et organisation des soins et services

Le temps d'attente à l'arrivé avant la prise en charge avait été jugé " court (<30 mn) » par $89,2 \%$ et « long (> $30 \mathrm{mn})$ » par 10,8\% des répondants. La qualité du service reçu était jugée bonne par $95,2 \%$ des clients. Ce même taux estimait avoir été soigné en toute sécurité. Un pourcentage de $4,8 \%$ de répondants n'étaient pas du tout satisfait des conditions hygiéniques du district sanitaire. Concernant les prestations bénéficiées 95,2\% des répondants estimaient être satisfaits (Tableau II).

\section{Mode de facturation des actes}

Nous avons trouvé que seulement $8,4 \%$ des répondants étaient sous système d'assurance et qu'ils étaient tous sous le système d'Assurance Maladie Obligatoire (AMO). Concernant le lieu d'obtention des médicaments prescrits moins de la moitié des répondants $(49,4 \%)$ affirmaient avoir acheté tous les médicaments prescrits au sein du district sanitaire. Un pourcentage non négligeable de $18,3 \%$ de répondants déclarait qu'on leur avait exigé de payer une caution, et la jugeaient tous moins humaine. S'agissant de la facturation $34,9 \%$ des répondants la jugeaient « Pas du tout abordable » (Tableau II).

\section{Discussion}

\section{Limites de l'étude et biais}

Les limites qui ont entravées ce travail sont d'abord la taille de l'échantillonnage qui est réduit. Cela est dû à l'insuffisance de ressource financière.

Nous avons opté pour une interview en face à face puisque plus de la moitié de nos patients $(51,81 \%)$ étaient non scolarisés. Or, si ces non scolarisés représentent une majorité dans notre population d'étude, ce sont justement ces personnes issues de couches défavorisées, qui sont susceptibles de présenter différents problèmes liés à la communication, à l'accès aux soins et donc présenter des spécificités en termes de satisfaction [4,5]. Ce mode d'interview offrait l'avantage de réduire le taux de non réponse et de données manquantes. Elle a par contre l'inconvénient de ne pas respecter l'anonymat et d'exposer au biais de désirabilité sociale qui pourrait surestimer la satisfaction. Ainsi, le patient exprimerait moins facilement son insatisfaction $[4,8]$. Cependant, pour limiter ce biais, l'enquêteur était quasiment étranger aux services et inconnu du personnel habituel des unités d'hospitalisation. Le patient ou l'accompagnant était en mesure de répondre au questionnaire sans risquer de mettre en difficulté sa relation avec le personnel soignant. Le contexte socioculturel des patients pourrait également constituer un biais. En effet, la santé retrouvée constituait déjà pour certains patients une source de satisfaction et influençait les réponses aux questions. Cela était perceptible chez les patients provenant des zones rurales. Ceux-ci étant à la recherche de l'amélioration de leur état de santé et n'ayant pas d'exigences concernant la qualité des soins. Les autres biais possibles pouvaient être le fait que le personnel de santé était au courant de l'étude. Donc, qu'il ait eu de manière consciente ou non, une adaptation de l'attitude du personnel de santé face aux patients. Conscient de l'importance des limites et biais nous pensons que d'autres études pourront approfondir ce travail initial pour une meilleure satisfaction des usagers du district sanitaire de Koutiala en particulier et des autres structures sanitaires similaires.

\section{Satisfaction globale}

Un total de $94 \%$ de répondants était globalement satisfait de leur hospitalisation. Ce résultat est supérieur d'une part au $75,5 \%$ de patients globalement très satisfaits par le service trouvé dans par Franclet A. [17], et d'autre part au score de $78.3 \%$ de patients globalement satisfaits, trouvé par Yameogo AR. \& al [5]. Par ailleurs ce résultat concorde avec ceux trouvés par L'ANEH dans son étude dans les 13 établissements hospitaliers du Mali qui variaient de $93 \%$ au CNOS à $55 \%$ à l'hôpital du Point G [16]. Des difficultés diverses existent dans ce genre d'études qualitatives, à commencer par le fait que lorsqu'on interroge un patient ou son accompagnant, le ressenti n'est pas identique, ce qui va par conséquent influencer sur le niveau de satisfaction. Néanmoins, ce niveau élevé doit être interprété avec précaution. Ce taux élevé de satisfaction pourrait s'expliqué d'une part par la différence entre la taille des échantillons qui était beaucoup plus élevé dans ses études que dans le nôtre et d'autre part par le fait que les patients reçus au district sanitaire de Koutiala provenaient majoritairement du milieu rural et pas ou peu scolarisé et liant le plus souvent leur satisfaction à l'amélioration de leur état de santé et non aux autres paramètres des soins.

La réalité ci-haut décrite a été trouvée lors de l'étude sur la satisfaction des patients hospitalisés en Suisse par Richard Eisler qui révèle que les personnes qui se considèrent en mauvaise santé au moment de l'enquête étaient globalement moins satisfaites de leur hospitalisation que les personnes qui déclaraient être en très bonne santé [18].

\section{Caractéristiques sociodémographiques}

II est communément admis que les variables sociodémographiques (âge, sexe, profession,...) sont liées à la satisfaction globale $[1,6,7,18]$, dans notre étude comme retrouvé par Adamou H. \& al [3], ils n'avaient pas d'influence sur la satisfaction globale (Tableau III).

\section{Accompagnement social reçu}

Dans notre travail comme dans d'autres études $[3,5,17,19]$, la plupart des répondants estimaient avoir reçu un bon accueil. L'accueil du patient et de sa famille demeurent un facteur primordial. C'est plus qu'un acte banal de la vie quotidienne. L'arrivée d'un malade dans un service de soins est particulière. C'est pour lui un temps fort, un moment d'imprégnation où il est sensible et vulnérable et où il a besoin de soutien. Par ailleurs contrairement à d'autres études $[3,5,17,19]$, nous 
n'avons trouvé aucune association statistiquement significative entre l'accueil et la satisfaction globale des clients ( $p=0,273$ ) (Tableau IV).

Aucun répondant n'a déclaré avoir été victime de discrimination. Ce résultat est contraire à celui trouvé par Y. Jaffré \& JP. Olivier de Sardan qui affirment que les soignants jugent les patients sur leur apparence, et qu'une discrimination s'opère au détriment des pauvres [20]. L'absence de victime ne saurait dire l'inexistence de discrimination au sein du district sanitaire de Koutiala, mais pourrait s'expliqué par le fait que la presque totalité de nos enquêtés à leur arriver étaient des cas d'urgence dont la prise en charge ne pouvait guère laisser place à une discrimination.

Bien que la confiance aux soignants ne suffise pas, car chaque patient a ses attentes et sa perception des services qui lui sont rendus. Elle est la pièce-maitresse de toute bonne relation. En plus, nonobstant le fait que l'accueil n'est pas directement lié à la confiance, mais un patient bien accueilli est à moitié guéri selon certains patients, donc contribue de manière indirecte à instaurer un climat de confiance. Notre étude retrouve une association significative entre le fait d'avoir confiance aux soignants et la satisfaction globale des clients $(p=0,002)$ (Tableau IV).

La presque totalité des répondants estimait bonne la collaboration entre ses proches et les soignants. Cette collaboration est très importante dans la mesure où le proche (familier) est impliqué dans la prise de décision concernant le malade surtout si son état ne lui le permet pas en plus des différentes démarches concernant la santé du patient à l'intérieur et en dehors de la structure de santé. II existe une relation significative entre l'existence d'une bonne collaboration entre les proches (familiers) et les soignants et la satisfaction globale des clients $(p=0,018)$ (Tableau IV).

Comme retrouvé par plusieurs auteurs $[3,5,17,19]$, notre étude retrouve une association significative entre le sentiment d'être traité en toute dignité et la satisfaction globale des clients $(\boldsymbol{p}=\mathbf{0 , 0 1 7 )}$ (Tableau IV). En effet, un patient estimant n'avoir pas été soigné de façon digne pourrait être difficilement satisfait des services qui lui sont rendus.

Répondre clairement aux questions du patient ou de ses proches est une dimension capitale dans l'accompagnement social, car elle permet de lever l'angoisse et le doute qui s'installent chez le patient et ses proches tout au long de son séjour. Notre analyse révèle une association très significative entre le fait d'avoir eu des réponses claires aux questions posées et la satisfaction globale des clients $\left(p=10^{-3}\right)$ (Tableau IV). Au district sanitaire de Koutiala $92,8 \%$ des répondants mettaient moins de cinq minutes à attendre les soignants en cas de besoin et estimaient raisonnable ce délai d'attente. Au CHB 51,6\% soutiennent que lorsqu'ils font appel à un soignant, le délai d'attente moyen est de 5 minutes [17]. Notre étude a trouvé une relation significative entre le délai d'attente en cas de besoin et la satisfaction globale des clients $(p=0,04)$ (Tableau IV).
La plupart des répondants ont déclaré pouvoir recommander le service à ses proches. Cela pourrait s'expliqué par le fait que les clients liaient le plus souvent l'intention de recommander à l'amélioration de leur état de santé et non aux autres paramètres des soins. Comme retrouvé par d'autres auteurs [3,5], notre étude retrouve que ceux qui disaient être insatisfait n'étaient pas disposés à recommander le district à un parent ou un proche $\left(p=10^{-3}\right)$.

\section{Prestations offertes et organisation des soins et services}

Le temps d'attente à l'arrivée avant la prise en charge a été jugé « court » par la plupart des répondants $(89,2 \%)$. Adamou H. \& al trouvent que le temps d'attente a été « jugé long » avant la prise en charge pour $41,41 \%$ des patients [3]. Cette différence pourrait s'expliqué par le fait que la majorité des hospitalisés étaient des cas d'urgences à leur arrivé nécessitant une prise en charge rapide.

Contrairement à l'étude faite au Niger [3], notre analyse n'a pas retrouvé d'association significative entre le temps d'attente avant la prise en charge et la satisfaction globale des patients $(\boldsymbol{p}=\mathbf{0 , 0 8 9 )}$ (Tableau IV).

La qualité du service était jugée bonne par la presque totalité des répondants $(95,2 \%)$. Au Niger seulement $54,63 \%$ étaient satisfaits de la prise en charge [3]. La guérison ou l'amélioration de l'état du patient est tributaire de la qualité du service reçu. Nos analyses révèlent une relation très significative entre la qualité du service et la satisfaction globale des patients $\left(p=10^{-3}\right)$ (Tableau IV). En effet $60 \%$ des clients qui disaient être insatisfait de la qualité des services reçu étaient globalement insatisfait.

Dans notre étude comme dans plusieurs autres [3,5,19], une relation significative existe entre la sécurité des soins reçu par les patients et la satisfaction globale $(p=0,017)$ (Tableau IV). La sécurité des soins est une dimension importante dans la prise en charge d'un malade surtout en ce temps de maladies infectieuses létales tel que le VIH, l'Hépatite B, le virus Ebola, le paludisme....

La disposition générale, le confort et la propreté des locaux sont autant de facteurs qui mettent en confiance le patient et qui sont liées à une meilleure satisfaction des usagers [3]. Dans ce travail une relation très significative existe entre les conditions hygiéniques du district sanitaire et la satisfaction globale des patients $(p=$ $10^{-3}$ ) (Tableau IV). En fait, tous les patients qui se disaient «Pas du tout satisfait» ou «Partiellement satisfait » des conditions hygiéniques étaient globalement insatisfaits.

Dans l'ensemble, les prestations offertes correspondaient aux attentes des clients. Dans notre étude comme retrouvé par Adamou $H$. \& al une association très significative existe entre la qualité des prestations offertes et la satisfaction globale des patients $\left(p=10^{-3}\right)$ (Tableau IV). Par ailleurs tous les clients qui n'appréciaient pas les prestations bénéficiées étaient globalement insatisfaits. 


\section{Mode de facturation des actes}

Plus du quart des répondants affirmaient qu'on leur avait exigé une caution. Ce taux bien que faible doit être interprété avec précaution. Les caractéristiques sociodémographiques des répondants pourraient constituer une barrière au fait de donner une réponse négative par crainte de ne pas nuire à sa relation avec les soignants. Nonobstant cela, de telles pratiques sont à bannir de nos structures sanitaires car elles sont de nature à décourager leur fréquentation. Contrairement à l'étude faite par Ntibenda RM. \& al [19], dans le nôtre une relation significative existe entre l'exigence d'une caution et la satisfaction globale des patients $(p=0,003)$ (Tableau IV). En effet 4 clients sur 5 ayant déclaré qu'on leur avait exigé une caution étaient globalement insatisfaits.

Même si aucune association significative n'existe entre le fait d'être sous un système d'assurance $(p=0,054)$, entre le lieu d'obtention des médicaments prescrits $(p=0,361)$, entre le coût de la facturation $(\boldsymbol{p}=1)$ (Tableau IV) et la satisfaction globale des patients, le coût de nos prestations sanitaire doivent être révisé, compte tenu du faible niveau de revenu de nos ménages qui n'ayant pas, pour la plupart, accès aux systèmes d'assurance supporte la majeur partie des dépenses sanitaires.

\section{Conclusion}

La plupart des patients était satisfaite des prestations et services rendus au district sanitaire de Koutiala. Notre étude a montré que la plupart des clients liaient leur satisfaction globale à l'amélioration de leur état de santé. Cependant une importance doit être accordée aux points d'insatisfactions car bien que faible, ils constituent des éléments importants pour l'amélioration de la qualité des soins et services.

\section{Conflits d'intérêt}

Les auteurs ne déclarent aucun conflit d'intérêt.

\section{Contribution des auteurs}

Tous les auteurs ont contribué à la correction du document, ils ont lu et approuvé la version finale du manuscrit.

\section{Remerciements}

Nos vifs remerciements s'adressent au corps administratif, médical, paramédical et aux clients du district sanitaire de Koutiala.

\section{Références}

1. Thi PLN, Lê TG, Empereur $F$, Briançon $S$. Satisfaction des patients hospitalisés à Hô Chi MinhVille, Viet Nam. Santé Publique. 2002;14(4):345-60.

2. Aurélien MR. Les fonctions de l'évaluation des services publics locaux par la satisfaction des usagers, entre apprentissage et discipline [Internet] [Thèse PhD]. [France]: Université d'Orléans; 2010 [cité 9 mai 2019]. Disponible sur: www.archivesouvertes.fr
3. Adamou H, Amadou M I, Habou O, Halidou M, Karimou S, Sani R, et al. Satisfaction des patients, élément important dans la globalité des soins : cas d'un service des urgences chirurgicales au Niger. Mali Médical. Tome 32. 2017;20-6.

4. Bougmiza I, Ghardallou ME, Zedini C, Lahouimel H, Nabli-Ajmi T, Gataa R, et al. Evaluation de la satisfaction des patientes hospitalisées au service de gynécologie obstétrique de Sousse, Tunisie. Pan African Medical Journal. 18 avr 2011;8:2.

5. Yameogo AR, Millogo GRC, Palm AF, Bamouni J, Mandi GD, Kologo JK, et al. Évaluation de la satisfaction des patients dans le service de cardiologie du CHU Yalgado Ouédraogo. Pan African Medical Journal. 2017;28:1-8.

6. Canoui-Poitrine $F$, Logerot $H$, Frank-Soltysiak $M$. Évaluation de la satisfaction des professionnels et des patients d'une unité multidisciplinaire de chirurgie ambulatoire. Pratiques et Organisations des Soins. 2008;39(4):323-30.

7. Cantin J, Rocheleau L. Sondage sur les attentes et la satisfaction des usagers hospitalisés à l'égard des services offerts à l'Hôpital Louis-H. Lafontaine [Internet]. Université de Montréal: Direction des soins infirmiers, Hôpital Louis-H. Lafontaine; 2006 [cité 23 mars 2019] p. 06 . Disponible sur: www.stat.gouv.qc.ca

8. Diouf M, Cisse D, Cmm L, Ginsburg FD, Traoré R. Évaluation de la satisfaction des patients admis en service d'odontologie à Dakar. Pratiques et Organisations des Soins. 2010;41(3):225-30.

9. Agence National d'Evaluation des Hôpitaux (ANEH). Evaluation de la mise en œuvre de la réforme hospitalière au Mali [Internet]. 2010 [cité 5 mai 2019]; Bamako, Mali. Disponible sur: www.aneh-mali.org

10. Le Ministre de la santé. Arrêté portant charte du malade dans les établissements hospitaliers au Mali [Internet]. 08-2716 / Ms-Sg oct 6, 2008 p. 03. Disponible sur: www.mail.cnom.santé.gov.ml

11. Président de la république du Mali. Loi 02-049 ANRM du 22 Juillet 2002 portant loi d'orientation sur la santé [Internet]. 2002 [cité 2 mai 2019]. Disponible sur: www.mail.cnom.sante.gov.ml

12. Président de la république du Mali. Loi 02-050 ANRM du 22 Juillet 2002 portant loi hospitalière [Internet]. 2002 [cité 2 mai 2019]. Disponible sur: www.mail.cnom.sante.gov.ml

13. Coulibaly S. Koutiala, la capitale de l'or blanc [Internet]. sekoulafia. 2009 [cité 22 mars 2019]. Disponible sur: sekoucoulibaly.unblog.fr

14. Bernabé BP. Le programme PECIMA de Helen Keller International (HKI) dans le district sanitaire de 
Koutiala. Mali; 2013 p. 6. Disponible sur: www.coverage-monitoring.org

15. Dolo $H$. Evaluation de l'état nutritionnel et de la mortalité chez les enfants de 0 à 59 mois dans le cercle de Koutiala (Mali) [Internet] [Thèse de médecine]. [Bamako, Mali]: Faculté de Médecine et d'Odontostomatologie; 2014 [cité 20 mars 2019]. Disponible sur: www.keneya.net

16. Agence National d'Evaluation des Hôpitaux (ANEH). Rapport de synthèse de la performance des Hôpitaux au titre de 2017 [Internet]. 2018 [cité 28 mars 2019]. Disponible sur: www.sante.gov.ml

17. Franclet $A$. Enquête de satisfaction auprès des usagers de la PASS de l'Hôpital Saint-André de Bordeaux. [Internet]. [Université de Bordeaux]: U.F.R. des Sciences Médicale; 2017 [cité 20 juin 2019]. Disponible sur: hal.archives-ouvertes.fr

18. Eisler R. La satisfaction des patients hospitalisés en Suisse [Internet]. Zurich; 2007 août [cité 28 juill 2019] p. 29. Disponible sur: www.comparis.ch

19. Mugisha Ntibenda R, Karafuli K, Omer K. Evaluation du niveau de satisfaction des usagers du centre hospitalier Bethesda de Goma en République démocratique du Congo. [Internet]. Mémoire Online. 2012 [cité 1 déc 2018]. Disponible sur: www.memoireonline.com

20. Jaffré $Y$, Olivier de Sardan J-P. Une médecine inhospitalière : Les difficiles relations entre soignants et soignés dans cinq capitales d'Afrique de l'Ouest. [Internet]. Abidjan, Bamako, Conakry, Dakar, Niamey; 2003 [cité 9 mai 2019]. 66-275 p. Disponible sur: www.cairn.info 
Tableau I: Répartition des répondants en fonction des caractéristiques sociodémographiques

\begin{tabular}{|c|c|c|c|}
\hline Variables & Modalités & Effectif $(\mathrm{N}=83)$ & $\%$ \\
\hline \multirow[t]{2}{*}{ Sexe } & Masculin & 32 & 38,6 \\
\hline & Féminin & 51 & 61,4 \\
\hline \multirow[t]{6}{*}{ Catégories d'âge } & $<18$ ans & 8 & 9,6 \\
\hline & $18-34$ ans & 34 & 41 \\
\hline & $35-44$ ans & 10 & 12 \\
\hline & $45-54$ ans & 10 & 12 \\
\hline & $55-64$ ans & 3 & 3,6 \\
\hline & $\geq 65$ ans & 18 & 21,7 \\
\hline \multirow[t]{4}{*}{ Provenance } & Commune urbaine de Koutiala & 29 & 34,9 \\
\hline & Commune rurale de Koutiala & 39 & 47 \\
\hline & Autres localités du Mali & 12 & 14,5 \\
\hline & Hors du Mali & 3 & 3,6 \\
\hline \multirow[t]{7}{*}{ Principale profession } & Cultivateur & 30 & 36,1 \\
\hline & Fonctionnaire & 4 & 4,8 \\
\hline & Commerçant(e) & 9 & 10,8 \\
\hline & Ménagère & 25 & 30,1 \\
\hline & Élève / Étudiant & 6 & 7,2 \\
\hline & Ouvrier & 7 & 8,4 \\
\hline & Autres (à préciser) & 2 & 2,4 \\
\hline \multirow{4}{*}{ Niveau de scolarisation } & Non scolarisé & 43 & 51,8 \\
\hline & Primaire & 19 & 22,9 \\
\hline & Secondaire & 20 & 24,1 \\
\hline & Supérieur (Universitaire) & 1 & 1,2 \\
\hline \multirow[t]{3}{*}{ Etat marital } & Célibataire & 12 & 14,5 \\
\hline & Marié(e) & 70 & 84,3 \\
\hline & Veuf(ve) & 1 & 1,2 \\
\hline
\end{tabular}

Tableau II: Répartition des répondants en fonction des dimensions explorés

\begin{tabular}{|c|c|c|c|c|}
\hline Variables & Modalités & Effectif & $\%$ & Effectif total \\
\hline \multirow[t]{2}{*}{ Qualité de l'accueil } & Oui & 78 & 94 & \multirow{2}{*}{83} \\
\hline & Non & 5 & 6 & \\
\hline \multirow[t]{2}{*}{ Confiance dans les soignants } & Oui & 77 & 92,8 & \multirow{2}{*}{83} \\
\hline & Non & 6 & 7,2 & \\
\hline \multirow{2}{*}{$\begin{array}{l}\text { Collaboration entre les proches (familiers) } \\
\text { et les soignants. }\end{array}$} & Oui & 76 & 95 & \multirow{2}{*}{80} \\
\hline & Non & 4 & 5 & \\
\hline \multirow{2}{*}{ Etre traiter en toute dignité } & Oui & 79 & 95,2 & \multirow{2}{*}{83} \\
\hline & Non & 4 & 4,8 & \\
\hline \multirow[t]{2}{*}{ Réponse claire aux questions posées } & Oui & 70 & 88,6 & \multirow{2}{*}{79} \\
\hline & Non & 9 & 11,7 & \\
\hline \multirow[t]{5}{*}{ Délai d'attente en cas de besoin } & Moins de 5 minutes & 77 & 92,8 & \multirow{5}{*}{83} \\
\hline & 6 à 10 minutes & 3 & 3,6 & \\
\hline & 11 à 15 minutes & 1 & 1,2 & \\
\hline & 16 à 20 minutes & 1 & 1,2 & \\
\hline & Plus de 20 minutes & 1 & 1,2 & \\
\hline \multirow{4}{*}{$\begin{array}{l}\text { Intention de recommander } \\
\text { le district sanitaire à un proche } \\
\text { Temps d'attente à l'arrivé }\end{array}$} & Oui & 78 & 94 & \multirow{2}{*}{83} \\
\hline & Non & 5 & 6 & \\
\hline & Court $(<30$ minutes $)$ & 74 & 89,2 & \multirow{2}{*}{83} \\
\hline & Long (> 30 minutes) & 9 & 10,8 & \\
\hline \multirow{2}{*}{ Qualité du service reçu } & Oui & 79 & 95,2 & \multirow{2}{*}{83} \\
\hline & Non & 4 & 4,8 & \\
\hline \multirow{2}{*}{ Sécurité des soins reçus } & Oui & 79 & 95,2 & \multirow[b]{2}{*}{83} \\
\hline & Non & 4 & 4,8 & \\
\hline \multirow{2}{*}{ Satisfait des prestations bénéficiées } & Oui & 79 & 95,2 & \multirow[b]{2}{*}{83} \\
\hline & Non & 4 & 4,8 & \\
\hline \multirow[t]{3}{*}{ Conditions hygiéniques } & Totalement satisfait & 71 & 85,5 & \multirow{3}{*}{83} \\
\hline & Partiellement satisfait & 8 & 9,6 & \\
\hline & Pas du tout satisfait & 4 & 4,8 & \\
\hline \multirow[t]{2}{*}{ Adhérant à un système de santé } & Oui & 7 & 8,4 & \multirow{2}{*}{83} \\
\hline & Non & 76 & 91,6 & \\
\hline \multirow{2}{*}{$\begin{array}{l}\text { Lieu d'approvisionnement en } \\
\text { médicaments prescrits }\end{array}$} & Ici & 40 & 49,4 & \multirow[b]{2}{*}{81} \\
\hline & Ici et dehors & 38 & 46,9 & \\
\hline
\end{tabular}


MALI SANTE PUBLIQUE 2019 SOMASAP

\begin{tabular}{llccc}
\hline \multirow{3}{*}{ Exigence de la caution } & Dehors & 3 & 3,7 & \\
& Oui & 15 & 18,3 & 82 \\
Appréciation de la facturation & Non & 67 & 81,7 & \\
& Très abordable & 7 & 8,4 & \\
& Abordable & 47 & 56,6 & 83 \\
& Pas du tout abordable & 29 & 34,9 & \\
\hline
\end{tabular}

Tableau III : Répartition des caractéristiques sociodémographiques en fonction de la satisfaction globale

\begin{tabular}{|c|c|c|c|c|c|}
\hline \multirow{2}{*}{ Variables } & \multirow{2}{*}{ Modalités } & \multirow{2}{*}{ Effectif total } & \multicolumn{2}{|c|}{ Satisfaction globale } & \multirow{2}{*}{$p$-value } \\
\hline & & & Non & Oui & \\
\hline \multirow[t]{2}{*}{ Sexe } & Masculin & \multirow{2}{*}{83} & 1 & 31 & \multirow{2}{*}{0,644} \\
\hline & Féminin & & 4 & 47 & \\
\hline \multirow[t]{2}{*}{ Age } & $\leq 35$ ans & \multirow{2}{*}{83} & 2 & 42 & \multirow{2}{*}{0,662} \\
\hline & $\geq 36$ ans & & 3 & 36 & \\
\hline \multirow[t]{2}{*}{ Provenance } & Commune urbaine de Koutiala & \multirow{2}{*}{68} & 3 & 26 & \multirow{2}{*}{0,644} \\
\hline & Commune rurale de Koutiala & & 2 & 37 & \\
\hline \multirow[t]{2}{*}{ Profession principale } & Cultivateur & \multirow{2}{*}{55} & 2 & 28 & \multirow{2}{*}{0,147} \\
\hline & Ménagère & & 1 & 24 & \\
\hline \multirow[t]{2}{*}{ Niveau de scolarisation } & Non scolarisé & \multirow{2}{*}{83} & 3 & 40 & \multirow[b]{2}{*}{1} \\
\hline & Scolarisé & & 2 & 38 & \\
\hline \multirow[t]{2}{*}{ Etat marital } & Célibataire & \multirow{2}{*}{82} & 1 & 11 & \multirow[b]{2}{*}{0,476} \\
\hline & Marié(e) & & 3 & 67 & \\
\hline
\end{tabular}

Tableau IV : Répartition des dimensions explorées en fonction de la satisfaction globale

\begin{tabular}{|c|c|c|c|c|c|}
\hline \multirow{2}{*}{ Variables } & \multirow{2}{*}{ Modalités } & \multirow{2}{*}{$\begin{array}{c}\text { Effectif } \\
\text { total }\end{array}$} & \multicolumn{2}{|c|}{ Satisfaction globale } & \multirow{2}{*}{$p$} \\
\hline & & & Non & Oui & \\
\hline \multirow[t]{2}{*}{ Qualité de l'accueil } & Oui & 83 & 4 & 74 & \multirow{2}{*}{0,273} \\
\hline & Non & 83 & 1 & 4 & \\
\hline \multirow[t]{2}{*}{ Confiance dans les soignants } & Oui & 83 & 2 & 75 & \multirow{2}{*}{0,002} \\
\hline & Non & & 3 & 3 & \\
\hline \multirow{4}{*}{$\begin{array}{l}\text { Collaboration entre les proches (familiers) } \\
\text { et les soignants. } \\
\text { Etre traiter en toute dignité }\end{array}$} & Oui & \multirow[b]{2}{*}{80} & 3 & 73 & \multirow{2}{*}{0,018} \\
\hline & Non & & 2 & 2 & \\
\hline & Oui & & 3 & 76 & \multirow{2}{*}{0,017} \\
\hline & Non & 83 & 2 & 2 & \\
\hline \multirow[t]{2}{*}{ Réponse claire aux questions posées } & Oui & & 0 & 70 & \multirow{2}{*}{$10^{-3}$} \\
\hline & Non & 19 & 5 & 4 & \\
\hline \multirow[t]{2}{*}{ Délai d'attente en cas de besoin } & Moins de 5 minutes & & 3 & 74 & \multirow[b]{2}{*}{0,040} \\
\hline & Plus de 5 minutes & 83 & 2 & 4 & \\
\hline \multirow{2}{*}{$\begin{array}{l}\text { Intention de recommander } \\
\text { le district sanitaire à un proche }\end{array}$} & Oui & & 1 & 77 & \multirow{2}{*}{$10^{-3}$} \\
\hline & Non & 83 & 4 & 1 & \\
\hline \multirow{2}{*}{ Temps d'attente à l'arrivé } & Court $(<30$ minutes $)$ & & 3 & 71 & \multirow[b]{2}{*}{0,089} \\
\hline & Long (> 30 minutes) & 83 & 2 & 7 & \\
\hline \multirow[t]{2}{*}{ Qualité du service reçu } & Oui & & 2 & 77 & \multirow{2}{*}{$10^{-3}$} \\
\hline & Non & 83 & 3 & 1 & \\
\hline \multirow[t]{2}{*}{ Sécurité des soins reçus } & Oui & & 3 & 76 & \multirow{2}{*}{0,017} \\
\hline & Non & 83 & 2 & 2 & \\
\hline \multirow[t]{2}{*}{ Satisfait des prestations bénéficiées } & Oui & & 1 & 78 & \multirow{2}{*}{$10^{-3}$} \\
\hline & Non & 83 & 4 & 0 & \\
\hline \multirow[t]{2}{*}{ Conditions hygiéniques } & Satisfait & & 0 & 71 & \multirow{2}{*}{$10^{-3}$} \\
\hline & Pas satisfait & 83 & 5 & 7 & \\
\hline Adhésion à un système de santé & Oui & 83 & 2 & 5 & 0.054 \\
\hline & Non & 83 & 3 & 73 & 0,054 \\
\hline Lieu d'approvisionnement en & Ici & 82 & 1 & 39 & \\
\hline médicaments prescrits & Ici et dehors & 82 & 4 & 38 & 0,360 \\
\hline Exigence de la caution & Oui & 82 & 1 & 66 & 0003 \\
\hline & Non & 82 & 4 & 11 & 0,003 \\
\hline Appréciation de la facturation & Abordable & & 3 & 51 & 1 \\
\hline & Pas du tout abordable & 83 & 2 & 27 & 1 \\
\hline
\end{tabular}




\section{Evaluation de la surveillance épidémiologique de la rougeole : Analyse de la base de données du district sanitaire} de Koulikoro de 2012-2018

\section{Evaluation of the epidemiological surveillance of measles: Analysis of the Koulikoro health district database from 2012-2018}

Coulibaly Hamadou' ${ }^{1}$ Sangho Oumar²,3, Sogodogo Seydou4, Keyembé Ken ${ }^{5}$, Dakouo Hyacinthe ${ }^{1}$

\author{
${ }^{1}$ Centre de santé de référence de Koulikoro, Mali \\ 2Département d'Enseignement et de Recherche en Santé \\ Publique, FMOS, USTTB, Mali \\ ${ }^{3}$ DER des Sciences Biologiques et Médicales FAPH, USTTB, \\ Mali \\ ${ }^{4}$ Inspection de la Santé, Ministère de la Santé et des Affaires \\ Sociales \\ ${ }^{5}$ AFENET (African Field Epidemiology Network),
}

Auteur correspondance : Dr Hamadou COULIBALY, Médecin généraliste, Epidémiologiste de terrain niveau intermédiaire, chargé de recherche au centre de santé de référence de Koulikoro, Mali ; Tél: (00223) 76453870 / 65791174; E-mail : drahmadouc@gmail.com / drhamadou_ml@yahoo.fr

\section{RESUME}

Introduction: La rougeole est une maladie éruptive fébrile, due à un virus (Morbillivirus). L'absence de résultats d'analyse sur la rougeole dans le district, nous a conduit à l'analyse des données de surveillance épidémiologique de la rougeole de 2012 à 2018, les objectifs étaient de décrire les caractéristiques de la maladie en temps lieux et personnes et d'identifier les facteurs associés à la survenue de la rougeole. Matériel et méthodes: Nous avons conduit une étude transversale, à l'aide des fiches de notifications, le cahier de déclaration, la liste linéaire et les fiches du laboratoire de 2012 à 2018, saisie et analyse sur Epi Info 7.2. Résultats : Dans notre étude, nous avons retrouvé $n=48$ cas suspects notifiés de 2012 à 2018 dont $6 \%$ des cas positif $(\lg \mathrm{M}+)$, le sexe masculin était le plus représenté $n=27$ (56) $P=0,38$, les sujets âgés de 15 ans et plus ont représenté la totalité des cas positifs $(\operatorname{lgM}) \mathrm{n}=3$ (100) $P=0,0002$, les sujets (suspects et confirmés) $n=6$ n'étaient pas vaccinés contre la rougeole $P=0,0002$, les cas suspects et confirmés $n=31(64,5)$ provenaient de la ville de Koulikoro. Conclusion: L'analyse de la base, nous a permis de retrouver trois cas suspects et confirmés dans le district. Les hommes étaient plus concernés que les femmes. Tous les cas avaient au moins 15 ans et aucun n'était vacciné contre la rougeole. Cette situation nécessiterait un renforcement de la vaccination de routine et une étude sur une plus longue période pouvant s'intéresser aux facteurs déterminants.

Mots clés: Analyse, base de données, rougeole, Koulikoro, Mali

\footnotetext{
ABSTRACT

Introduction: Measles is a febrile eruptive disease caused by a virus (Morbillivirus). The absence of measles analysis results in the district, led us to the analysis of measles epidemiological surveillance data from 2012 to 2018 , the objectives were to describe the characteristics
}

of the disease in time and person and to identify the factors associated with the occurrence of measles. Material and methods: We conducted a crosssectional study, using the notification forms, the declaration book, the linear list and the laboratory files from 2012 to 2018, entered and analyzed on Epi Info 7.2. Results: In our study, we found $n=48$ suspected cases from 2012 to 2018, of which 6\% were positive (lgM +), the male sex was the most represented $n=27(56) P=$ 0.38 , the elderly were 15 years and over represented all positive cases (lgM) $n=3(100) P=0.0002$. Subjects (suspected and confirmed) $n=6$ were not vaccinated against measles $P=0.0002$, the suspected and confirmed cases $n=31$ (64.5) came from the city of Koulikoro. Conclusion: The analysis of the database, allowed us to find three suspected and confirmed cases in the district. Men were more concerned than women. All cases were at least 15 years old and none were vaccinated against measles. This suggest a reinforcement of routine immunization and a study over a longer period that may be help to identify the determinant factors.

Keywords: Analysis, database, measles, Koulikoro, Mali

\section{Introduction}

La rougeole est une maladie éruptive fébrile, due à un virus de la famille des Paramyxoviridae (Morbillivirus) (1). Dans le monde entre 2000 et 2016, environ 20,4 millions de décès ont été évité par la vaccination contre la rougeole (2), qui fait de ce vaccin le meilleur investissement dans le domaine de la santé publique (3). En Afrique plusieurs pays ont enregistré des flambées épidémiques de la rougeole en 2018, et c'est Madagascar qui a enregistré 120000 cas avec environ 1000 décès (4). Certains pays ont ainsi procédé à l'évaluation de leur système de surveillance (5-7). La vaccination est l'un des moyens sures pour protéger les personnes contre la maladie (8). A l'opposé la faible couverture vaccinale accroit le taux de morbidité et de mortalité liées aux maladies évitables par la vaccination chez les enfants de moins de cinq ans et les cinq à quatorze ans, qui est accentuée dans certaines zones par des situations de crises sécuritaires et la famine (8). En 2018, le Mali a enregistré 1634 cas suspects de rougeole parmi lesquels 1408 ont été prélevés avec 476 positifs (IgM) (9). L'Organisation Mondiale de la Santé (OMS) recommande l'amélioration de la couverture vaccinale, le renforcement des systèmes de surveillance, la formation des agents de santé, l'amélioration de la qualité des données (10). Au Mali les principaux résultats montrent une amélioration dans les différents domaines 
de la stratégie d'amélioration de la couverture vaccinale (9).

Dans la région de Koulikoro en 2018, il a été enregistré 334 cas suspects dont 298 cas prélevés et 95 confirmés (IgM) (9). II a été noté la survenue d'épidémie ayant fait l'objet de riposte dans trois districts sanitaires durant cette même année, notamment Kati (Bacoumana et Dogodouma), Kangaba (10 aires de santé) et Kalabancoro (Kabala, et malgré que la couverture vaccinale anti rougeoleuse était de $98 \%$ (9).

La couverture vaccinale du district sanitaire de Koulikoro en 2018, était de $77 \%$ (9) qui est très largement inférieur au norme attendue $(\geq 95 \%)$. Selon les résultats de notre étude descriptive de l'analyse de la base de données de surveillance épidémiologique de la rougeole de 2012 à 2018, nous avons retrouvé 48 cas suspects de rougeole, tous prélevés avec 3 cas positifs (IgM) (11), et la dernière épidémie déclarée avec riposte remonte en 2013. L'importance de la maladie, l'efficacité du vaccin et la faiblesse de la couverture vaccinale dans le district sanitaire de Koulikoro, l'absence de résultats d'analyse antérieure de la base ont motivé la présente étude dont l'objectif est l'évaluation de la surveillance épidémiologique de la rougeole à travers l'analyse de la base de données du district sanitaire de Koulikoro de 2012-2018. Le but est de contribuer à l'amélioration du système de surveillance et de contrôle de cette maladie.

\section{Matériel et méthode}

II s'agissait d'une étude transversale. Nous avons procédé à une analyse des données de surveillance de la rougeole de 2012 à 2018 au niveau du centre de santé de référence de Koulikoro. Ont été exclus de l'étude les cas chez qui l'âge, le sexe et la provenance n'étaient pas spécifiés. Nous avons utilisés les fiches de notification, le cahier de déclaration des maladies, la liste linéaire des cas, les fiches de monitorage au niveau du laboratoire du CSRéf. Nous avons constitué une base de données sur Epi info 7.2. Nous avons réalisé des analyses descriptives et bivariées. Le test statistique utilisé a été le test exact de Fisher avec un seuil de significativité fixé à $5 \%$.

\section{Résultats :}

De 2012 à 2018, le district sanitaire de Koulikoro a notifié 48 cas suspects de rougeole dont trois sont revenus positifs (IgM) soit une incidence cumulée de 6\%. Parmi ces 48 cas suspects déclarés, seuls 13 soit $27 \%$ des prélèvements ont eu un résultat de laboratoire disponible. La totalité des cas avait été notifiée par les structures publiques notamment les Centres de Santé Communautaire (CSCOM) et le Centre de Santé de Référence de Koulikoro (CSRéf). Sur les 21 CSCOM fonctionnels que compte le district sanitaire de Koulikoro, 10 ont notifié (Figure 1) durant la période, de 2012 à 2018, concernée par la collecte des données d'où une proportion de $47,6 \%$.

Dans le cadre de cette surveillance nous avons constaté une moyenne de 6,8 cas suspects par an, avec une plus forte notification en 2016 avec 18 cas suspects, dont le seuil épidémique fixé à 5 cas suspects dans un mois a été dépassé au mois de mars 2016 (figure 2). Le sexe masculin était le plus représenté parmi les cas suspects avec $56,25 \%$. Les enfants de 0 à 5 ans ont été les plus représentés, le mode était de 5 ans et l'étendu entre 1-30 ans (figure 3). Dans l'analyse bivariée, les hommes avaient 1,58 fois plus de susceptibilité d'être atteints par la rougeole que les filles, mais la relation n'était pas significative $(p=0,38)$ (tableau I).

\section{Discussion}

De 2012 à 2018, le district sanitaire de Koulikoro a notifié 48 cas suspects de rougeole dont trois sont revenus positifs (IgM) avec une incidence de 6\%. Ces cas étaient tous âgés de 15 ans et plus, contrairement à la majorité des résultats de revues littéraires dans lesquelles les enfants de moins de 5 ans étaient les plus touchés $(5,12,13)$. Cela pourrait être expliqué par la faible proportion de disponibilité des résultats de $27 \%(\mathrm{~N}=48)$ à partir du laboratoire National de référence, à une insuffisance dans la qualité de la vaccination en termes d'atteinte des objectifs. L'analyse montre que le sexe masculin était le plus représenté avec $56,25 \%$ ( $N=48$ ). La même tendance a été rapportée par l'étude réalisée par Mitiku B. et al. en Ethiopie avec $65 \%(\mathrm{~N}=150)$ mais contraire à l'étude réalisée par Barry $\mathrm{D}$. et al à Sikasso avec 58,3\% ( $\mathrm{N=36}$ ) de sexe féminin (14). Dans notre étude la majorité des cas provenaient des zones urbaines contrairement à l'étude réalisée Jasem J. et al. en Irak ou les zones rurales étaient les plus représentées (13).

L'absence de vaccination est un déterminant majeur de la rougeole. Dans notre étude, aucun des trois cas n'était vacciné. Ce résultat est comparable à celui de l'étude réalisée par Farra A. et al., sur les données de 20072015 en République Centre Africaine (RCA), qui trouve une relation significative entre l'absence de vaccination et la survenue de la rougeole (15). En République Démocratique du Congo (RDC), la vaccination était un facteur significativement protecteur contre la rougeole avec un Rapport de Cotes (RC) ajusté à $95 \%$ de 0,20 $(0,15-0,26)(6)$. Nous n'avons pas pu avoir d'explication à la survenu de la rougeole chez les 15 ans et plus. Cependant la faiblesse de la couverture vaccinale était de $77 \%$ largement en deçà de la norme admise (9), cela pourrait avoir contribué à leur survenue comme trouvé dans l'étude du RDC (6).

Limite de l'étude : la taille de l'échantillon était faible mais surtout, aucun cas de rougeole n'était vacciné. Cela a impacté les analyses statistiques du RC. Cette taille ne permet pas de bien conclure du point de vue statistique. En outre la moitié des CSCOM (11/21) ainsi que les structures privées n'ont fait aucune notification durant toute cette périodes. Ils sont considérés comme muets dans le cadre de la surveillance et impact la taille de l'échantillon mais aussi la qualité de cette surveillance. 


\section{Conclusion}

L'analyse de la base, nous a permis de retrouver 48 cas suspects dont trois confirmés dans le district sanitaire de Koulikoro. Les hommes étaient plus concernés. Tous les cas avaient au moins 15 ans et aucun n'était vacciné contre la rougeole. Ceci implique un renforcement de la vaccination de routine et une étude sur une plus longue période pouvant s'intéresser aux facteurs déterminants.

\section{Références}

1. Médecins sans frontières. Prise en charge d'une épidémie de rougeole, Guide pratique à l'usage des médecins, infirmiers, techniciens de laboratoire, auxiliaires de santé et logisticiens. 2013.

2. Dabbagh A. Progress Toward Regional Measles Elimination - Worldwide, 2000-2017. MMWR Morb Mortal Wkly Rep [Internet]. 2018 [cité 13 juill 2019];67. Disponible sur: https://www.cdc.gov/mmwr/volumes/67/wr/mm6747a6.ht $\mathrm{m}$

3. OMS | Nouvelles données de surveillance de la rougeole pour 2019 [Internet]. WHO. [cité 20 juin 2019]. Disponible sur:

http://www.who.int/immunization/newsroom/measlesdata-2019/fr

4. Debela MB, Kahsay AB, Mokonnon TM, Shifaw ZG. Evaluation of Measles Surveillance Systems in Afar Region, Ethiopia: A Descriptive Evaluative Study, 2017. 5. Ameh CA, Sufiyan MB, Jacob M, Waziri NE, Olayinka AT. Evaluation of the Measles Surveillance System in Kaduna State, Nigeria (2010-2012). Online J Public Health Inform. 2016;8(3):e206.

6. Doshi RH, Mukadi P, Shidi C, Mulumba A, Hoff NA, Gerber S, et al. Field evaluation of measles vaccine effectiveness among children in the Democratic Republic of Congo. Vaccine. 26 juin 2015;33(29):3407-14.

7. Masresha B, Katsande R, Luce R, Fall A, Shibeshi M, Weldegebriel G, et al. Performance of National Measles Case-Based Surveillance Systems in The WHO African Region. 2012 - 2016. J Immunol Sci. 2 août 2018;Suppl:130-4.

8. Ibrahim BS, Usman R, Mohammed Y, Datti Z, Okunromade $\mathrm{O}$, Abubakar AA, et al. Burden of measles in Nigeria: a five-year review of casebased surveillance data, 2012-2016. Pan Afr Med J [Internet]. 22 janv 2019 [cité 30 août 2019];32(Suppl 1). Disponible sur: https://www.ncbi.nlm.nih.gov/pmc/articles/PMC6445333/ 9. DNS Annuaire Système Local d'Information Sanitaire 2018 VF du 27 avril.pdf [Internet]. [cité 25 juin 2019]. Disponible sur:
http://www.sante.gov.ml/docs/AnnuaireSLIS2018VFdu27 avril.pdf

10. OMS. Elimination de la rougeole d'ici 2020 stratégies pour la région africaine. 2011.

11. DRS Koulikoro. Base de données de surveillance épidémiologique. 2018.

12. Debela M, Bayray A, Marama T, Shifaw Z. Evaluation of Measles Surveillance Systems in Afar Region, Ethiopia: A Descriptive Evaluative Study, 2017. Int J Biotech Trends Technol. 18 janv 2019;9.

13. Jasem J, Marof K, Nawar A, Monirul Islam KM. Epidemiological analysis of measles and evaluation of measles surveillance system performance in Iraq, 20052010. Int J Infect Dis IJID Off Publ Int Soc Infect Dis. mars 2012;16(3):e166-171.

14. Barry D, Traoré B. Evaluation du système de surveillance de la rougeole dans le district sanitaire de Sikasso. 2016 p. P25.

15. Farra A, Loumandet TN, Pagonendji M, Manirakiza A, Manengu C, Mbaïlao R, et al. Epidemiologic profile of measles in Central African Republic: A nine year survey, 2007-2015. PLOS ONE. 20 mars 2019;14(3):e0213735. 
Liste des tableaux et figures :

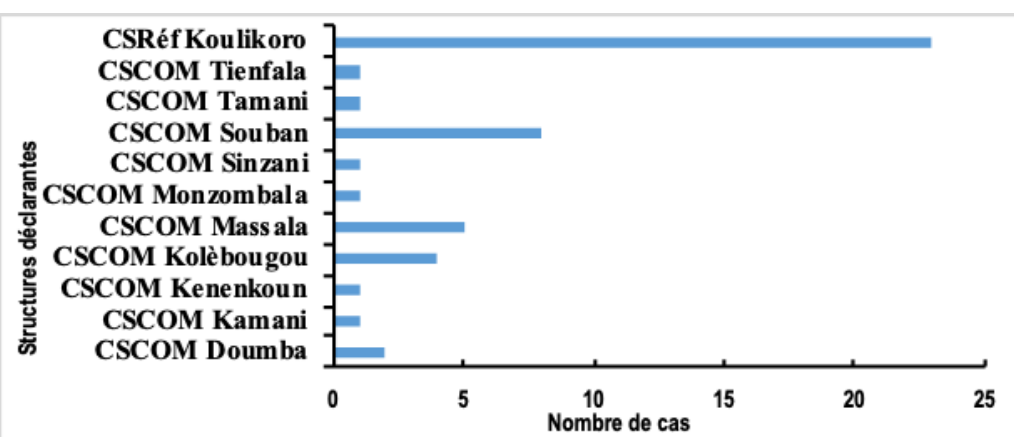

Figure 1 : Nombre de cas suspects de rougeole notifiés, par structure, district sanitaire de Koulikoro 2012-2018

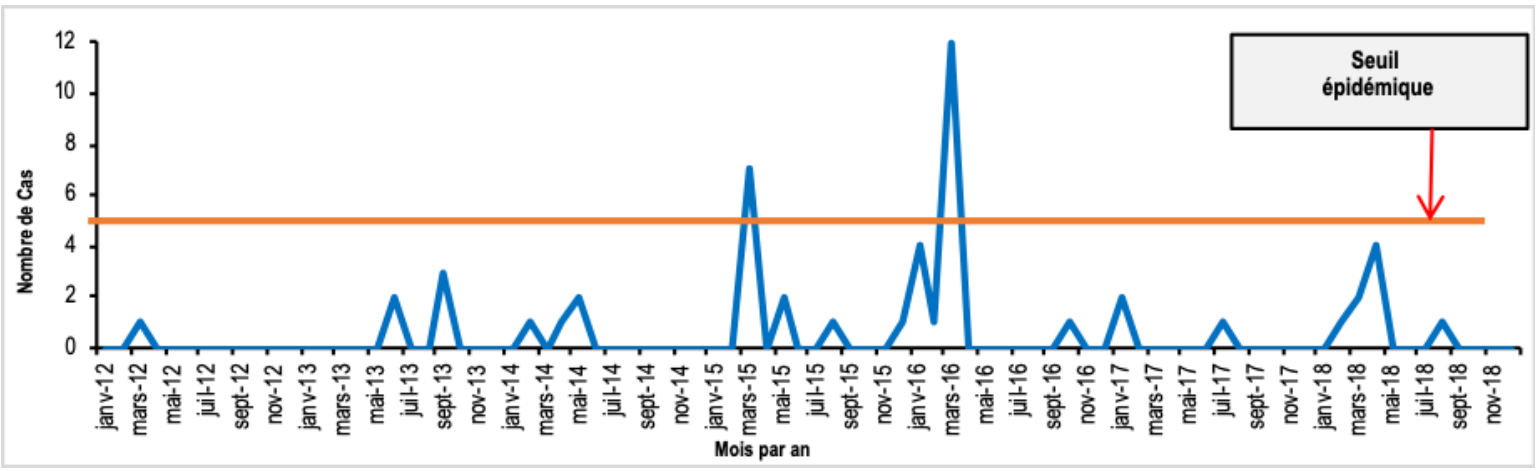

Figure 2 : Tendance des cas suspects de rougeole par mois et année, district sanitaire de Koulikoro, 2012 - 2018

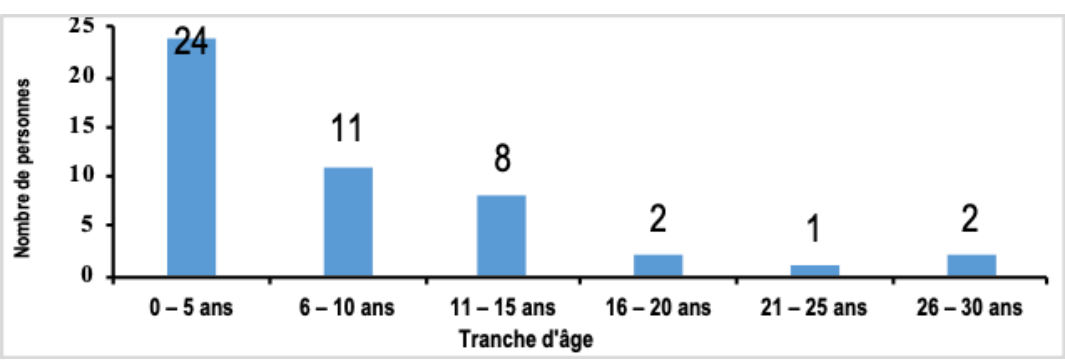

Figure 3 : Répartition des cas suspects de rougeole par tranche d'âge, district sanitaire de Koulikoro, 2012-2018

Tableau I : relation entre les facteurs, sexe, statut vaccinal, résidence, tranche d'âge et la rougeole dans le district sanitaire de Koulikoro, 2012-2018

\begin{tabular}{lcccc}
\hline \multicolumn{1}{c}{ Variables } & Rougeole Oui $(\mathrm{N}=3)$ & Rougeole Non (N=45) & RC [IC95\%] & $\mathbf{P}$ \\
\hline Sexe & 2 & 25 & $1,58[0,11-49,42]$ & 0,38 \\
Masculin & 1 & 20 & & \\
$\quad$ Féminin & 0 & 17 & $0[0,0-3,10]$ & 0,12 \\
$\begin{array}{l}\text { Statut vaccinal } \\
\text { Vacciné }\end{array}$ & 3 & 28 & & \\
Non vacciné & 2 & 29 & $1,10[0,07-34,48]$ & 0,48 \\
Résidence & 1 & 16 & & \\
Urbaine & & & & \\
Rurale & 0 & 43 & $0,0[0,0-0,14]$ & 0,0002 \\
Tranche d'âge & 3 & 2 & & \\
Moins de 15 ans & 0 & & & \\
15 ans et Plus & 3 & & & \\
\hline
\end{tabular}




\title{
Epilepsie liée aux accidents de la voie publique chez les Guinéens en 2012
}

\author{
Epilepsy linked to road accidents among Guineans in 2012
}

\author{
Diarra $\mathrm{AS}^{1}$, Dansoko DD¹, Bocoum FA¹, Guilavogui $\mathrm{V}^{2}$, Beavogui $\mathrm{KL}^{2}$.
}

\begin{abstract}
1 Département d'Etude et de Recherche en Santé Publique du Mali 2 Hôpital National Donka, service de neurochirurgie, BP 234 Conakry Guinée

Correspondant : Dr Diaba Denise DANSOKO s/c DERSP, Tel : 6699 34 82, diabadenise@gmail.com
\end{abstract}

\section{Résumé}

Introduction : L'épilepsie post-traumatique est la survenue d'une ou plusieurs crises épileptiques au-delà de la première semaine suivant un traumatisme crânien. Les séquelles invalidantes qu'engendrent cette pathologie, les difficultés de prise en charge, l'augmentation des accidents de la voie publique ont motivé notre étude. Son but était d'étudier la place des accidents de la voie publique dans les épilepsies pour contribuer à l'amélioration de sa prise en charge et la sécurité routière en Guinée en 2012. Méthode : C'était une étude transversale de Janvier 2010 à Décembre 2012 qui a porté sur 21 dossiers de malades hospitalisés pour traumatisme cranio encéphalique ayant développé une EPT. Les données du questionnaire ont été analysées sur Epi Info version 3.5.1, les iconographies interprétées par un spécialiste. Résultat : La prévalence de l'affection était de 1,6\% parmi les hospitalisés (21/1304) avec une prédominance masculine $66,7 \%$ et un sex ratio de 2. Les accidents de la voie publique étaient les circonstances de survenue les plus importantes avec $81 \%$. A l'admission $90,5 \%$ des patients avaient un trouble de la vigilance, $52,4 \%$ un Traumatisme crânien sévère, $57,1 \%$ une crise tonico-clonique, $61,9 \%$ un point d'impact frontal, $85,7 \%$ une anomalie focalisée à l'électroencéphalogramme et $95,2 \%$ ont eu une amélioration de l'état clinique avant la sortie. Conclusion L'élément préventif sûr de l'Epilepsie Post Traumatique reste la lutte contre les traumatismes crâniens en insistant sur l'éducation routière, en impliquant les médias dans la stratégie de sensibilisation et en améliorant les conditions de sécurité routière.

Mots clés: Epilepsie post traumatique, traumatisme crânien, Guinée

\footnotetext{
Abstract:

Introduction: Post-traumatic epilepsy is the occurrence of one or more epileptic seizures beyond the first week following a head trauma. The disabling sequelae caused by this pathology, the difficulties of treatment, the increase in accidents on the public highway motivated our study. Its aim was to study the place of accidents on the public highway in epilepsy to contribute to the improvement of its management and road safety in Guinea in 2012. Method: It was a cross-sectional study from January 2010 to December 2012 which focused on 21 files of patients hospitalized for cranio encephalic trauma having developed EFA. The questionnaire data were analyzed on Epi Info
}

version 3.5.1, the iconographies interpreted by a specialist. Result: The prevalence of the disease was $1.6 \%$ among hospitalized patients $(21 / 1304)$ with a male prevalence of $66.7 \%$ and a sex ratio of 2 . Accidents on the public highway were the most important circumstances with $81 \%$. On admission $90.5 \%$ of patients had an alertness disorder, $52.4 \%$ had a severe head trauma, $57.1 \%$ had a tonic-clonic attack, $61.9 \%$ had a frontal impact point, $85.7 \%$ a focused anomaly on the electroencephalogram and $95.2 \%$ had an improvement in the clinical state before discharge. Conclusion The sure preventive element of Post Traumatic Epilepsy remains the fight against head trauma by insisting on road education, by involving the media in the awareness raising strategy and by improving road safety conditions.

Keywords: Post traumatic epilepsy, head trauma, Guinea

\section{INTRODUCTION :}

L'épilepsie post-traumatique (EPT) est la survenue d'une ou plusieurs crises d'épilepsie au-delà de la première semaine suivant un traumatisme crânien (TC).

Pour affirmer EPT, il faut que le TC précède les crises mais aussi qu'il en soit la cause.

Les crises précoces de moins de $24 \mathrm{H}$ ou retardées de plus d'une semaine sont des crises symptomatiques aigues correspondant à la réaction du cerveau aux effets physiques du TC. Les crises secondaires ou tardives survenant après la première semaine répondent à la définition de la maladie épileptique post traumatique (1).

Dans le monde, les études épidémiologiques retrouvent que l'EPT représente $20 \%$ des épilepsies symptomatiques dans la population générale et $5 \%$ en consultation spécialisée d'épilepsie (2).

L'incidence de l'EPT est difficile à apprécier à cause de la très grande diversité de la notion TC. Dans les séries (populations) militaires, on observe des proportions concordantes de 30 à $40 \%$ en cas de plaies craniocérébrales (3). Dans les séries (populations) civiles, en raison notamment de la rareté des plaies craniocérébrales, l'incidence est nettement plus faible, de l'ordre de 3 à $7 \%$, voire moins si l'on inclut les TC bénins dans les statistiques (4).

En Afrique, le traumatisme crânien constitue la cause de l'épilepsie chez $14 \%$ des enfants de moins de 14 ans, 30\% des jeunes âgés de 15 à 34 ans, et $8 \%$ des adultes de plus de 65 ans (5).

Les facteurs de risque responsable de l'épilepsie post traumatique sont les plaies crânio-cérébrales, les embarrures associées à des hématomes extra ou intracérébraux, les lésions focales intracérébrales et l'amnésie post traumatique supérieure à $24 \mathrm{~h}$. 
En fonction de la gravité du traumatisme crânien, l'incidence cumulative de l'épilepsie est de 2,1\% après un TC léger ; $4,2 \%$ après un TC modéré et de $16,7 \%$ après un TC sévère (5).

En Guinée, les TC constituent un problème majeur de santé publique du fait de leur fréquence, leur gravité, et surtout de leurs séquelles lourdes. Les accidents de la voie publique (AVP) restent la première cause des TC dans toutes les catégories de gravité (6). Les accidents de la voie publique entrainent très souvent des épilepsies soit immédiates ou tardives et qui passent souvent inaperçues dans les services de santé.

Les séquelles invalidantes qu'engendrent cette pathologie, sa stigmatisation, les difficultés de prise en charge, l'augmentation des accidents de la voie publique et le besoin de ralentir ce fléau ont motivé notre étude. Elle avait pour but d'étudier la place des accidents de la voie publique dans la survenue des épilepsies afin de contribuer à l'amélioration de la sécurité routière et de la prise en charge de l'EPT à l'hôpital de Donka en Guinée. C'est dans ce cadre que la présente étude «Epilepsie liée aux accidents de la voie publique chez les Guinéens en 2012 » a été initiée.

\section{MATERIEL METHODES :}

Notre étude transversale s'est déroulée dans le service de Neurochirurgie de l'hôpital national Donka de janvier 2010 à 31 décembre 2012 et a porté sur 21 dossiers des malades hospitalisés pour TCE qui ont développé une EPT.

Matériels utilisés : le registre d'hospitalisation, les dossiers des malades et une fiche d'enquête.

Taille de l'échantillon : recrutement exhaustif des dossiers de tous les patients vus pour traumatisme crânien durant les 3 ans de l'étude soit 21 cas /1304 admissions en neurochirurgie.

Ont été inclus dans l'étude tous les patients de tout âge, de tout sexe, et toute provenance ayant développé au moins deux (2) crises épileptiques après le 7 ème jour suivant leur TC sans ou avec traitement antiépileptique.

Des crises survenues lors des troubles métaboliques, Tous dossiers incomplets (types de crises, mode de survenue, le traitement)

Les tests statistiques descriptives comme les comparaisons (Khi 2), les calculs de fréquence et moyenne ont été faits.

Les informations recueillies ne permettront pas de remonter à l'identité des malades car nous n'avons utilisé que les dossiers des patients avec l'approbation de l'hôpital de Donka. La confidentialité a été gardée sur les dossiers exploités.

RESULTATS :
L'enquête a concerné 21 dossiers de malades hospitalisés pour traumatisme cranio encéphalique ayant développé une Epilepsie Post Traumatique.

Les AVP ont constitué les circonstances de survenue les plus importantes $(81 \%$ dont $52,4 \%$ par Moto, $19 \%$ automobile) représentant $71,4 \%$ des EPT (tableau I). Nous avons reçu $52,4 \%$ des patients avec un Trauma crânien sévère.

L'étude a montré une prédominance masculine $66,7 \%$ avec un sex ratio de 2

Les élèves et étudiants étaient les plus touchés avec $47,6 \%$ (tableau II).

La prévalence de l'EPT était de $1,6 \%$ dans la population hospitalisée et les patients avaient un âge compris entre 2 et 65 ans avec une médiane de 24,59 ans.

A l'admission $90,5 \%$ des patients avaient un trouble de la vigilance, $57,1 \%$ une crise tonico-clonique (tableau III), $61,9 \%$ un point d'impact frontal (tableau IV) et $85,7 \%$ une anomalie focalisée à l'EEG.

La totalité des patients a bénéficié d'un traitement médical et $95,2 \%$ ont eu une amélioration de leur état clinique avant la sortie.

Dans notre étude $52,4 \%$ des patients ont eu un intervalle du séjour de 15 à 30 jours

\section{DISCUSSION}

Les limites principales de ce travail ont été l'inaccessibilité financière de certains patients à l'imagerie par résonnance magnétique, à l'EEG / scanner cérébral et l'insuffisance de suivi des dossiers.

Dans notre étude $81 \%$ des patients ont fait une Epilepsie après un AVP dont 71,4\% par automobile et moto. Ces chiffres pourraient s'expliquer d'une part par le non-respect du code de la route, la non utilisation de ceinture de sécurité par les usagers ainsi que leur imprudence et d'autre part par le manque de panneau de signalisation sur les routes et leur dégradation. Ceci est concordant avec les résultats de Richard I et coll. (9) au service de rééducation fonctionnelle de CHR Nantes /France en 1998.

Le TC sévère après un AVP était de $52,4 \%$. Cette fréquence élevée serait probablement due à la violence du choc pendant l'accident et à la petite taille de notre échantillon. Ce résultat est discordant avec Gueguen B et coll. (10) qui trouve $11,5 \%$ pour le traumatisme crânien grave après un AVP.

La prédominance masculine à $66,7 \%$ s'expliquerait par la nature des activités menées par les hommes les exposant surtout aux AVP et blessures par arme à feu.

Ces résultats concordent avec DIALLO TM et coll. (7) en Guinée en 2004 qui trouvent 25 hommes contre 17 femmes, Richard I et coll. (9) rapportent 75 hommes contre 15 femmes, Wafae El et coll. (5) au Maroc en 2012 démontrent que les EPT dues aux AVP surviennent chez les adolescents et adultes jeunes de 15 à 25 principalement chez les hommes.

Notre étude a trouvé que les élèves létudiants ont été les plus touchés $47,6 \%$ probablement dû à l'exposition de cette couche aux AVP qui sont les étiologies les plus probables des EPT dans la population civile. 
La prévalence de l'EPT était à 1,6\% dans la population hospitalisée pourrait s'expliquer par la courte durée d'hospitalisation des patients ne permettant pas à un nombre plus élevé de patients de développer l'EPT en milieu hospitalier. DIALLO TM et coll. (7) en Guinée en 2004 rapportent 7,1\%, Garga N et coll. (8) en 2006 et Wafae EL (5) au Maroc en 2012 trouvent 20\% d'EPT dans la population générale.

Le trouble de la vigilance lié aux AVP était présent chez $90,5 \%$ des patients car les conducteurs à deux roues ne portent généralement pas de casque, ceux du véhicule pas de ceinture de sécurité et la tête est la plus exposée lors des AVP. Ces résultats sont approuvés par plusieurs auteurs Wafae El et coll. [(5) démontrent que l'incidence de l'EPT augmente de 15 à $30 \%$ selon que le coma est initial, quel que soit sa profondeur et dure une semaine \pm 3 semaines. Gueguen B (11) au centre hospitalier Sainte Anne de Paris en 2009 trouve le coma comme meilleur facteur prédictif d'EPT, qui fait augmenter son incidence de $15 \%$ si le coma dure plus de trois semaines.

Nous avons répertorié $57,1 \%$ cas de crises tonicocloniques nettement supérieur à celui de DIALLO TM et coll. (7) en Guinée en 2004 qui rapportent $35,7 \%$ de crises tonico-cloniques.

Le point d'impact le plus représenté était la région frontale avec $61,9 \%$ qui serait due à l'exposition de cette région lors des AVP d'une part, et son caractère très épileptogène d'autre part. Ce résultat est contraire à celui de Gueguen $B$ (10) qui rapporte une incidence plus élevée de la région pariétale que celle frontale ou temporale.

L'EEG a représenté $85,7 \%$ des bilans en imagerie due à son intérêt dans le diagnostic, la prise en charge, et le suivi de l'EPT. Ce résultat est similaire à celui de Tatai $\mathrm{Al}$ (11) en Algérie en 2012 qui trouve 89\% d'EEG comme examen réalisé sur 100 cas.

Nous avons eu une nette amélioration de l'état clinique avant la sortie chez $95,2 \%$ des patients pouvant s'expliquer par la mise en route du traitement médical bien conduit et/ ou chirurgical d'urgence dès l'admission. La littérature parle de $70 \%$ des épilepsies contrôlées par le traitement médical (12).

\section{CONCLUSION :}

L'EPT est une complication fréquente après un TC sévère et les accidents de la voie publique occupent une grande place dans sa survenue. Le traitement antiépileptique chez un traumatisé crânien en période aiguë peut éviter les complications mais inutile pour prévenir la survenue d'une épilepsie. L'élément préventif sûr de l'Epilepsie Post Traumatique reste la lutte contre les Traumatismes crâniens en insistant sur l'éducation routière, en impliquant les médias dans la stratégie de sensibilisation et en améliorant les conditions de sécurité routière.

Nos remerciements aux responsables de l'hôpital de Donka en Guinée, au personnel enseignant du DERSP du Mali. Mention spéciale au professeur Akory AG IKNANE qui nous a appris la rédaction scientifique / Directeur de l'Institut de Santé Publique du Mali.
Aucun conflit d'intérêt n'est lié au présent travail

\section{REFERENCES :}

Thivard L. Unité d'épileptologie et service de soins de suite et réadaptation neurologique du Pr Baulac, la Salpetrière, Paris ; 2004-12.

2. Maillard L, Michael S, Raffo E, Ducrocq $X$, Vespignani $\mathrm{H}$. Complications traumatiques des crises épileptiques Epilepsie volume 14 Numéro 147-54 Mars 2002.

3. Weiss G H ET COLL. Arch Neurol 1983; 40: 7-10.

4. Annerger J F et coll. Neurology $1980 ; 30: 683-689$.

5. Wafae EL, Nissrine L, Kissani N, Said Ait ben A L'épilepsie post traumatique à Marrakech dans North African and middle east epilepsy journal volum 1 number 2 march- april 2012

6. Dramou b. Profil des cas de TCE et VM dans le service de neurochirurgie de Donka 2001

7. Diallo TM, cisse A, Morel Y, Cisse AF, Souare IS. Premières crises épileptiques tardives étude de 42 cas méd trop 2004.

8. Garga N, Daniel HL L'épilepsie post-traumatique : un problème majeur en besoin urgent d'avancées majeures, L'épilepsie Curr 2006 Janvier, 6 (1): 1-5.

9. Richard I ET coll. Ann Réadaptation Med physique 1998, $41: 409-415$

10. Gueguen B. Epilepsie Post-Traumatique Centre Hospitalier Sainte-Anne Paris S. Guillou révue française 2009

11. Tatai AI Profil de l'épilepsie à Bechar en Algérie étude d'une cohorte de 100 cas dans North African and middle east epilepsy journal volum1 number2 march- april 2012

12. www.who.int/mentalheath/media/en/80/pdf

Liste des tableaux :

Tableau I : Répartition des patients accidentés selon la circonstance de survenue de l'accident à l'hôpital de Donka en 2012

\begin{tabular}{lcc}
\hline Circonstance de survenue & Nombre & $\%$ \\
\hline Accident domestique & 1 & 4,8 \\
Agression physique par arme à feu & 1 & 4,8 \\
AVP auto & 4 & 19,0 \\
AVP moto & 11 & 52,4 \\
AVP vélo & 2 & 9,5 \\
Chute & 2 & 9,5 \\
Total & 21 & 100 \\
\hline
\end{tabular}

Tableau II : Répartition des patients selon leur profession

\begin{tabular}{lcc}
\hline Profession & Nombre & $\%$ \\
\hline Elèves /Etudiants & 10 & 47,6 \\
Fonctionnaire & 1 & 4,8 \\
Profession libérale & 8 & 38,1 \\
Autre & 2 & 9,5 \\
Total & 21 & 100 \\
\hline
\end{tabular}

Autre : ménagère et sans profession 
Tableau III : Répartition des patients accidentés selon le type de crises à l'hôpital de Donka en 2012

\begin{tabular}{lll}
\hline Type de crise & Nombre & $\%$ \\
\hline Clonique & 2 & 9,5 \\
Tonico-clonique & 12 & 57,1 \\
Tonique & 2 & 9,5 \\
Myoclonique & 1 & 4,8 \\
Association & 4 & 19,0 \\
\hline
\end{tabular}

Tableau IV : Répartition des patients accidentés selon le point d'impact du trauma crânien à l'hôpital de Donka en 2012

\begin{tabular}{lll}
\hline Point d'impact du TC & Nombre & $\%$ \\
\hline Frontal & 13 & 61,9 \\
Temporal & 5 & 23,3 \\
Pariétal & 5 & 23,3 \\
Occipital & 3 & 13,3 \\
\hline
\end{tabular}


Etude des cas de morsures de chiens de janvier 2017 à octobre 2019 dans le district Sanitaire de Sikasso, Mali

Case study of dog bites from January 2017 to October 2019 in the Sanitary District of Sikasso, Mali

Kampo $\mathrm{O}^{1}$, Traore $\mathrm{B}^{2}$, Sangho $\mathrm{O}^{3}$, Diakite $\mathrm{S}^{4}$, Telly $\mathrm{N}^{5}$

(1) Centre de Santé de Référence

Sikasso, BP 333 Sikasso, Mali. Tél.

: 002232225002

(2) AFENET (African Field

Epidemiology Network),

(3) Département d'Enseignement et

de Recherche en Santé Publique,

FMOS, USTTB

(4) Direction Régionale de la santé

de Sikasso

(5) 3Département d'Enseignement

et de Recherche en Santé

Publique, FMOS, USTTB

\section{Auteur correspondance : $\mathrm{Dr}$ \\ Oumar Kampo, Médecin \\ généraliste, Epidémiologiste de \\ terrain niveau intermédiaire, chargé \\ de recherche et de la surveillance \\ épidémiologique au centre de santé \\ de référence de Sikasso, Mali ; Tél: \\ (00223) 76115190 / 66770699; E- \\ mail : baroukampo@yahoo.fr}

\begin{abstract}
Résumé
Introduction : Au Mali, de 2015 à 2018, on a recensé 2611 cas de morsures de chiens dont 6 cas de rage humaine confirmés. Durant la même période, les cas de morsures de chiens à Sikasso, s'élèvent à 236 et est en constante augmentation d'année en année. Les objectifs étaient de décrire les cas de morsures en personne, Caractériser les lésions des morsures et apprécier la prise en charge des cas. Méthodologie: Nous avons mené une étude descriptive portant sur les dossiers des sujets mordus par chiens de janvier 2017 à octobre 2019 dans le District Sanitaire de Sikasso. Résultats : Les consultants recensés au nombre de 196 en majorité de sexe masculin (64\%), avaient un âge moyen de 14 ans. Seuls $31 / 196$, soit $15,81 \%$ des chiens étaient vaccinés. Les fesses ont été le site privilégié des morsures de chiens avec $39,80 \%$. Le schéma vaccinal à 5 doses avec lavage de la plaie représentait 52,52\%. Parmi les 196 consultants recensés, $15,62 \%$ avaient abandonné le traitement vaccinal. Les chiens mis en observation étaient $41,34 \%$ et un grand nombre abattu soit $38,26 \%$. La classification finale des cas fait état de trois (3) cas de rage canine et aucun cas de rage humaine. Conclusion La lutte contre la rage doit mettre l'accent le traitement post exposition précoce, la vaccination des animaux ainsi que sur la sensibilisation de la population vis-à-vis du risque de rage.
\end{abstract}

Mots clés : morsure, chien, vaccination, rage, Sikasso, Mali

\begin{abstract}
Introduction: In Mali, from 2015 to 2018, there were 2611 cases of dog bites, including 6 confirmed cases of human rabies. During the same period, cases of dog bites in Sikasso amounted to 236 and is constantly increasing year after year. Methodology: We conducted a descriptive study of the records of dog bitten subjects from January 2017 to October 2019 in the Health District of Sikasso. The objectives were to describe cases of bites in person, characterize bite injuries and assess case management. Results: The majority of the 196 consultants identified were male ( 64 per cent) and had an average age of 14 years. Only $31 / 196$ or $15.81 \%$ of the dogs were vaccinated. The buttocks were the preferred site of dog bites with $39.80 \%$. The 5 -dose vaccination regimen with wound cleansing accounted for $52.52 \%$. Of the 196 consultants surveyed, $15.62 \%$ had discontinued the vaccination regimen. Dogs put under observation were $41.34 \%$ and a large number slaughtered at $38.26 \%$. The final classification of cases showed three (3) cases of canine rabies and no cases of human rabies. Conclusion: Rabies control should focus on early postexposure treatment, vaccination of animals and raising public awareness of the risk of rabies.
\end{abstract}

Key words: dog bite, vaccination, rabies, Sikasso, Mali

\section{Introduction}

La rage est une zoonose virale provocant une encéphalo-myélite mortelle à $100 \%$, due à un Lyssavirus transmise par les carnivores domestiques (chien, chat) et sauvages (chauve-souris) à travers leur salive lors des morsures ou d'égratignures (1). C'est un problème de santé très préoccupant. Dans le monde, on compte 59.000 décès/ an lié à la rage dont $95 \%$ en Afrique et en Asie. C'est aussi dans ces deux continents que plus de 3 milliards de personnes sont potentiellement exposées à la rage $[2,3,4,5]$.

Les enfants de moins de 15 ans représentent $40 \%$ des personnes mordues par un animal $[6,2)]$.

Selon les rapports annuels de la Direction Nationale des services Vétérinaire et du laboratoire Central Vétérinaire du Mali, de 2015 à 2018, on a recensé 2611 de morsure de chiens. Avec 6 cas de rage humaine confirmés [5]. Sur 98 prélèvements effectués et analysés, nous avons obtenu $76,53 \%$ de cas positifs à la rage canine.

Devant l'augmentation des cas de morsure de chiens de plus en plus grave, nous avons initié cette étude qui a pour objectifs de décrire les morsures de chiens en personne; de décrire les lésions de morsures, d'apprécier la prise en charge des cas dans le District Sanitaire de Sikasso. 


\section{Méthodologie}

Cette étude descriptive s'est déroulée du 20 septembre au 20 octobre 2019 dans le district Sanitaire de Sikasso. Elle a concerné les données de la période du 01 janvier 2017 au 30 Octobre 2019

\section{Critères d'inclusion}

Toutes les personnes mordues par un chien pendant la période et tous les chiens mordeurs.

\section{Critère de non inclusion}

Toutes les morsures par un chien non renseignées dans les supports pendant la période d'étude dans le District Sanitaire.

\section{Collecte des données:}

Elle a été faite à partir des registres de prise en charge des patients mordus par animaux, les fiches de notification. Les informations recueillies étaient relatives aux caractéristiques sociodémographiques des patients (sexe, âge, profession, lieu d'habitation), la description des lésions (aspect ou type de lésion, le siège de la lésion et le mode de survenue); le traitement reçu (lavage des lésions, vaccin antirabique et l'immunoglobuline antirabique), devenir du patient et du chien mordeur.

\section{Echantillonnage}

Nous avons procédé à un choix raisonné.

\section{Taille de l'échantillon}

Notre étude a porté sur tous cas de morsures de chiens durant la période soit 196 cas.

Les données ont été collectées sur Excel et analysées sur le logiciel Epi7.2.

\section{Résultats}

De janvier 2017 à octobre 2019, 196 personnes ont été mordues par des chiens et ont été reçues dans les structures de santé du District Sanitaire de Sikasso avec une prévalence de $196 / 672872$ soit 29 pour 1000.000 habitants.

L'âge moyen des sujets était de 14 ans avec des extrêmes de 2 à 68 ans. Les moins de 15 ans représentaient $65 \% \%$ des cas. La tranche d'âge la plus touchée était celle de 5-9 ans (Figure 1).

La sex-ratio était de 1,77 en faveur des hommes. La majorité des sujets soit $71 \%$ provenait du milieu urbain et les attaques étaient provoquées dans $53 \%$ des cas et l'animal mordeur était de type domestique dans $(64,79 \%)$.

Les morsures de chiens ont été essentiellement localisées aux fesses et membres inférieurs avec respectivement $(38 \%)$ et $(33 \%)$ (Tableau III). Nous n'avons retrouvé que $15,87 \%$ des chiens vaccinés chez l'ensemble des animaux mordeurs mis en observation (41\%) (Tableau IIIII).

La prise en charge selon le protocole de Zagreb (schéma à 5 doses) associé aux soins de la morsure a été respectivement de $52,25 \%$, et $86,15 \%$. L'abandon du traitement était de 15,62 \% (Tableau IV).

La classification finale des cas fait état de 3 cas de rage canine et aucun cas de rage humaine durant la période d'étude.

\section{Discussion}

Cette étude comporte des limites. II a été impossible de lier certains animaux mordeurs à un ou à plusieurs sujets mordus du fait de l'absence de traçabilité dans les supports. Tous les animaux mordeurs n'ont pu être mis en observation et une fraction des suspects gardés et morts en observation n'ont pu être prélevée pour la confirmation au laboratoire central vétérinaire de Bamako. Cependant, les résultats obtenus ont permis d'atteindre nos objectifs.

Cependant, les résultats obtenus ont permis de voir l'ampleur des morsures de chiens dans le District Sanitaire de Sikasso. De janvier 2017 à octobre 2019, quelque 196 patients ont été victimes de morsures de chiens dans le District Sanitaire. Les hommes étaient les plus touchés soit $64 \%$ avec un Sex ratio (homme/femme) de 1,77. Cette prédominance masculine a été rapportée par Sylla K au Sénégal avec 66,4\% [7]. Nos résultats sont différents par contre de ceux rapportés par Marta $D$ $M$ [6] qui ont noté une prédominance féminine de $53 \%$. Face aux animaux, les hommes prennent-ils plus de risque que les femmes? Ou bien s'agit-il d'une imprudence de leur part? En effet, l'exposition au risque de transmission de la rage est le plus souvent la conséquence d'interactions comportementales dangereuses entre l'animal et la victime. La prédominance infantile des cas de morsures $(65 \%)$ chez les moins de 15 se retrouve chez beaucoup d'auteurs $[6,7,8]$. Chez l'enfant il s'agit généralement d'accidents domestiques et provoqué $(92 \%$ des cas. Cela pourrait s'expliquer par leur imprudence et méconnaissance face aux dangers de la rage. Un peu plus de la moitié des cas de morsures sont survenue en milieu urbain (61\%). Des résultats similaires ont été rapportés à Bamako par Dao $S$ et al [(8)] Ceci peut s'expliquer par la présence d'importantes décharges d'ordures, de gares routières et surtout de nombreuses gargotes. La forte densité de la population d'une part et la présence de nombreux chiens errants d'autre part, pourraient aussi expliquer le nombre élevé de victimes en milieu urbain. Les populations des centres urbains sont plus sensibilisées et informées au risque rabique que celles des régions. Ce qui justifie un recours rapide à une structure sanitaire en cas de morsure de chien.

Les morsures de chiens ont été essentiellement localisées aux fesses et membres inférieurs avec respectivement (38\%) et (33\%) ce qui est similaire avec l'étude de DAO S [8] qui trouva 60,8 \% aux membres inférieurs. Ceci pourrait s'expliquer par le fait que les animaux en pourchassant les victimes ont plus accès aux fesses et membres inferieurs qu'aux autres parties du corps.

Seuls 31 chiens soit $15,81 \%$ des chiens mordeurs étaient correctement vaccinés contre la rage et ce, malgré des multiples campagnes de vaccination Nationales des animaux de compagnies initiées par le pays depuis cinq années. Ce faible taux est rapporté par la plus parts des auteurs d'étude sur la rage en Afrique de façon générale 
$[7,9,10]$. On note également une fréquence élevée des chiens errants qui représentaient $39,53 \% \%$ des cas. Les animaux mis en observation représentaient $61 \%$. Ce faible taux peut s'expliquer par la méconnaissance de ce processus par la population qui, par peur de représailles et de poursuite, a tendance à abattre systématiquement les chiens mordeurs $(38,26 \%)$, associé à la fuite et disparition des chiens errants après forfait. Le pourcentage de personnes mordues misent sous traitement avec lavage de la plaie est de $52,25 \%$, sans lavage de la plaie, elle est d'environ 86,15\%. L'abandon du traitement était de $15,62 \%$. Le pourcentage d'abandon est largement inférieur à ceux trouvés par Tiembré [11] en côte d'ivoire en 2009 et $\mathrm{K}$ Sylla [7] au Sénégal qui sont respectivement de $46,9 \%$ et de $59 \%$. Ce problème d'inobservance au traitement incombe à la fois aux malades, mais aussi aux agents de santé qui prennent en charge les sujets exposés. Le manque d'information sur la maladie et son évolution ainsi que le cout élevé du traitement est un facteur important d'inobservance du traitement [12].

Au décours de cette étude, la manifestation clinique de la rage a été observée chez trois chiens dont un décès survenu en cours d'observation. Deux (2) prélèvements ont été effectués durant la période de l'étude avec un rendu du résultat positif. Ce qui nous à amener à classifier trois (3) cas de rage canine. Aucun cas de rage humaine n'a été notifié durant la période.

\section{Conclusion}

Aucun humain n'a déclaré la rage au cours de notre étude, par contre nous avons recensé trois cas de rage canine dans le District Sanitaire. Les enfants étant les plus touchés, il convient de mettre un accent sur la vaccination des animaux de compagnie qui est à l'origine de la presque disparition de la rage en occident [13 14, 15]. II convient de sensibiliser la population à la vaccination des animaux de compagnie qui selon beaucoup d'auteurs réduirait de façon significative le risque de transmission de la rage, $[3,10,16]$.

\section{Références}

1. Rotiyel Y. La rage_Importance actuelle en santé publique. In: Annales de l'Institut

Pasteur/Actualites. Elsevier; 1995. p. 100-109.

2. Rotivel Y, Goudal M, de Fanti AS. Prophylaxie de la rage humaine en France. Médecine Mal Infect. 2001;31:193-201.

3. Haddad N, Bourhy $\mathrm{H}$. La rage animale: risques autochtones et d'importation, mesures à prendre. Rev Francoph Lab. 2015;2015(472):35-49.

4. Ricard C, Thélot B, Sarcey G, Béata C, Servas $V$. Épidémiologie des morsures de chien en France: mise en place d'une enquête multicentrique mai 2009-avril 2010. Médecine ThérapeutiquePédiatrie.

2009;12(4):195-199.

5. Akakpo AJ. Le chien dans la societé noire africaine: un réservoir de rage. In: Rabies in the tropics. Springer; 1985. p. 516-519.

6. Rotivel Y, Goudal M, Wirth S, Tsiang H. Le risque de rage chez l'enfant qui voyage. Arch Pédiatrie. 1998;5(5):561-567.

7. Diop SA, Sow MS, Baldé MS, Diallo MOS, Bah

I, Cissé MO, et al. K Sylla.

8. Dao S, Abdillahi AM, Bougoudogo F, Toure K, Simbe $C$. Aspects épidémiologiques de la rage humaine et animale en milieu urbain à Bamako, Mali. Bull Soc Pathol Exot. 2006;99(3):183-186.

9. Bah SO, Chamoiseau G, Biha MLO, Fall

SMOA. Un foyer de rage cameline en Mauritanie. 1981;

10. Exposition à un risque d'infection rabique à

l'unité de prévention du service de maladies infectieuses et tropicales du CHNU de Fann à Dakar: Caractéristiques et prise en charge thérapeutique | Revue Malienne d'Infectiologie et de Microbiologie. [cité 11 nov 2019]; Disponible sur:

http://www.revues.ml/index.php/remim/article/view/1303

11. Tiembré I, Aka-Kone DMB, Konan YE, Vroh JBB, Ekra KD, Aka J, et al. Observance du traitement vaccinal antirabique chez les sujets exposés à la rage à Abidjan (Côte d'Ivoire). Santé Publique. 2009;21(6):595603.

12. Connaissances, attitudes et pratiques des chefs de ménage de la commune d'Abobo (Abidjan, Côte d'Ivoire) en matière de rage, en 2008 | Cairn.info [Internet]. [cité 18 déc 2019]. Disponible sur: https://www.cairn.info/revue-sante-publique-2014-4page-547.htm

13. Rotivel Y, Goudal M, de Fanti AS. Prophylaxie de la rage humaine en France. Médecine Mal Infect. 2001;31:193-201.

14. Bögel K, Meslin F-X. Aspects économiques de l'élimination de la rage humaine et canine: principles pour l'orientation des programmes. Bull World Health Organ. 1990;68(4):409.

15. Bouchrit N, Khyatti M, Nourlil J, Dardari R, Ibrahimy S, Tordo N, et al. Déterminants de la rage humaine au Maroc: variabilité génétique, qualité du vaccin ou prise en charge insuffisante. Médecine Mal Infect. 2002;32(9):508-513.

16. Rakotomalala W, Rakotonjanabelo AL, RakotoAndrianarivelo M, Roux JF, Zeller HG. La rage humaine à Madagascar (1996-1997). Arch Inst Pasteur Madagascar. 1998;64(1). 
LISTE DES TABLEAUX :

Tableau IV : Répartition des cas de morsures de chiens selon leur circonstance de survenu dans le district sanitaire de Sikasso de 2017-2019

\begin{tabular}{lllll}
\hline \hline $\begin{array}{l}\text { Circonstances } \\
\text { d'attaque }\end{array}$ & $\begin{array}{l}\text { Chiens } \\
\text { domestiques }\end{array}$ & $\begin{array}{l}\text { Chiens } \\
\text { errants }\end{array}$ & Total & $\%$ \\
\hline \hline provoquée & 43 & 61 & 104 & 53,00 \\
spontanée & 84 & 8 & 92 & 47,00 \\
Total & 127 & 69 & 196 & 100,00 \\
\hline \hline
\end{tabular}

Tableau II : Répartition des cas de morsures de chiens en fonction de la localisation des lésions.

\begin{tabular}{lll}
\hline \hline Localisation de la morsure & fréquence & $\%$ \\
\hline \hline Cou & 1 & 0,50 \\
Dos & 4 & 2,04 \\
Epaule & 1 & 0,50 \\
Fesses & 78 & 39,80 \\
Membre inférieurs & 60 & 30,61 \\
Membres supérieurs & 45 & 22,96 \\
Tête & 2 & 1,02 \\
Tronc & 5 & 2,55 \\
TOTAL & 196 & 100,00 \\
\hline \hline
\end{tabular}

Tableau III : Répartition des cas de morsures de chiens en fonction des mesures de contrôle et de prévention prises contre les chiens mordeurs

\begin{tabular}{lll}
\hline \hline variables & Effective & $\%$ \\
\hline \hline Mise en observation & 81 & 41,34 \\
Abattus & 75 & 38,26 \\
Disparus & 40 & 20,40 \\
Total & 196 & 100,00 \\
\hline \hline
\end{tabular}

Tableau IV : Répartition des cas de morsures de chiens en fonction de la prise en charge

\begin{tabular}{lll}
\hline \hline Variables & Effectif & $\%$ \\
\hline \hline Lavage des lésions + 5 doses Verrorab & 103 & 52,52 \\
Absence de traitement & 73 & 37,24 \\
Abandon du traitement (moins de 5 & 20 & 10,24 \\
doses) & 196 & 100,00 \\
Total & \\
\hline \hline
\end{tabular}




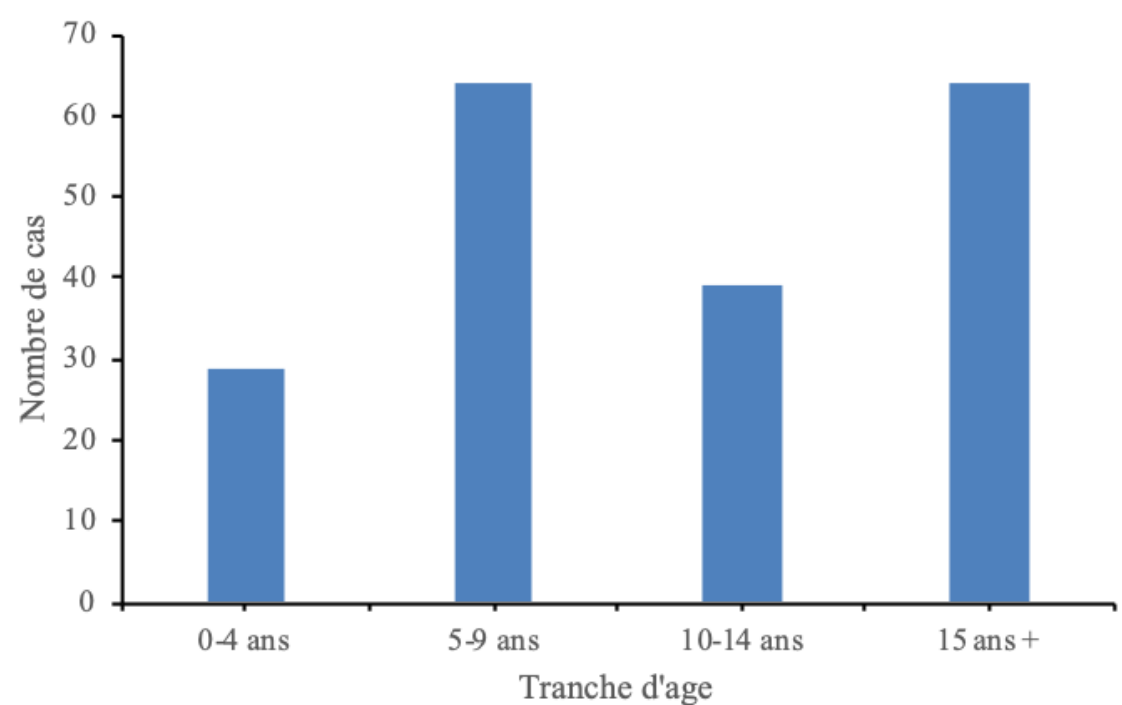

Figure1 : Répartition des morsures de chiens selon la tranche d'âge dans le District Sikasso de 2017-2019 
Rage au Mali, analyse des données de surveillance de la rage animale de 2009 à 2018

Rabies in Mali, analysis of animal rabies surveillance data from 2009 to 2018

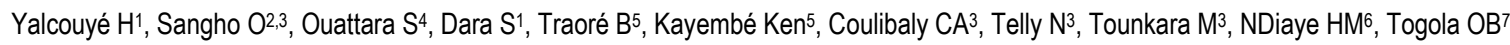

1 : Direction Nationale des Services Vétérinaires, Bamako, Mali 2 : DER des Sciences Biologiques et Médicales, FAPH/USTTB, Mali

3 : Département d'Enseignement et de Recherche en Santé Publique, FMOS/USTTB, Mali

4 : Direction Régionale de la Santé de Bamako, Mali

5 : Réseau Africain des Epidémiologiste de Terrain, AFENETMali, DGSHP, Bamako, Mali

6 : Direction Régionale de la Santé et de L'Hygiène Publique, Ségou, Mali

7 : Centre de Santé de Référence de Tominian, Ségou, Mali

Auteur correspondant : Dr Hamidou Yalcouyé, Direction Nationale des Services Vétérinaires, Bamako, Mali, 669726 23, 744882 62, yalcouye sn@yahoo.fr,

\section{Résumé}

Introduction: La rage est une maladie infectieuse, inoculable, provoquée par un virus du genre Lyssavirus. La contamination à l'homme se fait par morsure ou griffure par un chien enragé. Les laboratoires sont un élément essentiel de la surveillance de cette maladie. L'objectif de cette étude était d'analyser les données de la base de données de la rage au Mali de 2009 à 2018. Méthodes : II s'agissait d'une étude descriptive du 6 au 30 juin 2019. Nous avons réalisé une revue documentaire et exploité des données de la Base Access des résultats envoyées au Laboratoire Central Vétérinaire (LCV) par la Direction Nationale des Services Vétérinaires (DNSV). Les données de 10 ans, du 1er janvier 2009 au 31 décembre 2018, ont été exportées sur Excel 2013 et analysées sur Epilnfo7. Le Khi2 de Pearson ou le test Exact de Fisher ont été utilisés pour tester la liaison entre les variables. Résultats: La moyenne d'âge des chiens mordeurs était de 2,78 ans. Les mâles étaient plus fréquents avec $81,86 \%$. Les chiens étaient surtout du milieu urbain $(72,55 \%)$. L'incidence de la rage était de $39,92 \%$. Les chiens mordaient plus en saison sèche $(61,27 \%)$. L'agressivité $(74,02 \%)$ était le signe le dominant, suivi par la fureur avec $57,35 \%$. La fréquence de la vaccination chez les chiens de notre étude était $4,41 \%$. Conclusion : L'incidence de la rage était de $39,92 \%$. Les mâles étaient beaucoup plus impliqués dans la contamination, qui se faisait plus saison sèche. La fréquence de la vaccination était très faible. L'étude suggère l'intensification des mesures de vaccination des chiens.

Les mots clés : Rage animale, surveillance, Mali

Abstract

Introduction: Rabies is an infectious, inoculable disease caused by a virus of the genus Lyssavirus. Human contamination is by biting or scratching by a rabid dog. Laboratories are an essential part of surveillance for this disease. The objective of this study was to analyze data from the rabies database in Mali from 2009 to 2018. Methods: This was a descriptive study from June 6 to 30, 2019. We carried out a documentary review and used data from the Access Base of the results sent to the Central Veterinary Laboratory by the National Directorate of Veterinary Services. The 10-year data, from January 1, 2009 to December 31, 2018, were exported to Excel 2013 and analyzed on Epilnfo7. Pearson's Chi-square or Fisher's Exact test were used to test the link between the variables. Results: The average age of biting dogs was 2.78 years. Males were more common with $81.86 \%$. The dogs were mostly urban $(72.55 \%)$. The incidence of rabies was $39.92 \%$. Dogs bit more in the dry season $(61.27 \%)$. Aggression (74.02\%) was the dominant sign, followed by fury with $57.35 \%$. The frequency of vaccination in dogs in our study was $4.41 \%$. Conclusion: The incidence of rabies was $39.92 \%$. The males were much more involved in the contamination, which was done more dry season. The frequency of vaccination was very low. The study suggests stepping up vaccination measures for dogs.

Keywords: animal, rabies, surveillance, Mali

Introduction

La rage est une maladie infectieuse provoquée par un virus du genre Lyssavirus (1). Les animaux à sang chaud constituent le réservoir de virus (2). L'homme pouvant être contaminé, il s'agit d'une zoonose (2). La durée d'incubation de la rage est habituellement de 1 à 3 mois, mais peut s'étendre de moins d'une semaine à 1 an, en fonction des facteurs tels que le site de pénétration du virus et la charge virale (2).

La rage est une zoonose majeure présente sur les quatre continents (3). L'Afrique et l'Asie sont les Régions les plus affectées. Cette maladie sévit sur toute l'étendue du territoire malien.

La plupart des cas de rage est causée par des chiens et les enfants constituent la couche la plus touchée (1). La contamination se fait principalement par morsure ou pénétration de salive infectée dans une lésion ou une plaie ouverte, ou encore dans une muqueuse, au niveau de la bouche, de la cavité nasale ou des yeux (2). Des cas de contamination par inhalation du virus ont été démontrés, par exemple dans des grottes fortement peuplées de chauves-souris (4). De 1968 à 2018, 42 chiens et 3 chats ont été diagnostiqués atteints de rage en France (5). Tous ces cas ont été importés (5). L'apparition de ces cas a donné lieu à des prises en charge des personnes exposées allant de 2 à 187 personnes (selon le déplacement de l'animal (5). Dans 99\% des cas chez l'homme, la transmission se fait par le contact avec les chiens domestiques lors d'une morsure, griffure ou léchage (3). 
Selon l'Office International des Epizooties (OIE qui est l'Organisation Mondiale de la Santé Animale), près de 59 000 (cinquante-neuf mille) personnes meurent de suite de rage dans le monde chaque année (3). Selon l'OMS, $40 \%$ des victimes sont des enfants de moins de 15 ans (2). Les laboratoires sont un élément essentiel de la surveillance des maladies (6). Le coût de traitement d'une seule personne mordue par un chien peut servir à vacciner 25 chiens contre la rage. Le schéma classique de l'OMS comporte cinq injections intramusculaires dans l'épaule aux jours J0, J3, J7, J14, J30 et un rappel facultatif à $\mathrm{J} 90$.

La rage est inscrite sur la liste des maladies réputées légalement contagieuses sur le territoire de la République du Mali et sa surveillance est basée sur les textes législatifs et réglementaires dont la Loi $n^{\circ} 01-022$ du 31 mai 2001 régissant la répression des infractions à la police sanitaire des animaux sur le territoire de la République du Mali (7), le décret $n^{\circ} 01-339 / P-R M$ du 9 août 2001 fixant les modalités d'application de la loi n01-022 du 31 mai 2001 portant répression des infractions à la police sanitaire des animaux sur le territoire de la République du Mali (7) et le Décret n06412/P-RM du 27 septembre 2006 portant modification du décret $n^{\circ}$ 01-339/P-RM du 9 août 2001(7).

La notification de la maladie à l'OIE est obligatoire (8). Elle fait également partie de la liste des zoonoses priorisées dans le cadre du Global Health Security Agenda (GHSA, Agenda de Sécurité Sanitaire Mondial) et est inscrite sur la liste des maladies prioritaires du réseau de surveillance épidémiologique vétérinaire du Mali (Epivet-Mali). La rage est confirmée dans toutes les régions du Mali, la principale source de la maladie reste le chien enragé. Chaque année, des centaines de cas de morsures de chiens sur les hommes et d'autres animaux sont enregistrées (9). Les investigations qui s'en suivent (mise en observation, analyse d'échantillons) permettent d'établir un diagnostic. L'objectif de cette étude était de déterminer l'incidence et les facteurs déterminants de la rage.

\section{Méthode}

CADRE DE L'ETUDE : Le Laboratoire Central Vétérinaire (LCV) est un établissement public à caractère administratif qui est le laboratoire de référence en santé animale du Mali et à ce titre reçoit tous les échantillons pour suspicion de rage canine. Tout animal atteint de rage est immédiatement abattu. Les chiens et chats ainsi que tout autre mammifère sensible, mordus, roulés ou ayant été en contact avec l'animal enragé sont aussitôt abattus à l'exception des chiens qui ont été vaccinés préventivement depuis moins d'un an à condition qu'ils soient revaccinés dans les sept jours qui suivent la morsure, qu'ils restent sous surveillance. Les prélèvements sont effectués par les vétérinaires du secteur public comme ceux du privé et acheminés au LCV soit directement soit par voie hiérarchique (poste vétérinaire-secteur vétérinaire-DRSV-DNSV-LCV). Tous les résultats sont communiqués à la DNSV qui assure la retro information jusqu'au niveau de la base constituée par les Postes vétérinaires en passant par les Secteurs vétérinaires et les Directions régionales.

TYPE ET PERIODE D'ETUDE : II s'agissait d'une étude descriptive qui s'est déroulée du 06 au 30 juin 2019. Nous avons analysé les données enregistrées au LCV du 1 er janvier 2009 au 31 décembre 2018

POPULATION D'ETUDE : Tous les cas confirmés de rage animale.

TECHNIQUES DE COLLECTE DES DONNEES :

La technique a consisté à faire une revue documentaire et les données saisies sur Access ont été extraites sur Excel 2013. La qualité des données extraites a été vérifiée avec les fiches de prélèvement et de résultats archivées

TRAITEMENT ET ANALYSE DES DONNEES : Les données ont été nettoyées avec suppression des données aberrantes et incomplètes. L'analyse a été essentiellement descriptive avec Microsoft Excel et épi info 7. Les variables quantitatives ont été présentées par leur moyenne et leur écart type, les variables qualitatives par leurs nombres et proportions. Nous avons réalisé des analyses croisées entre variables qualitatives avec utilisation du Khi Deux de Pearson ou du test exact de Fisher avec un seuil de significativité de 0,05. Les résultats ont été présentés sous formes de textes, de tableaux et de figure.

CONSIDERATION ETHIQUE : Dans le cadre de cette étude aucun contact n'a été fait directement avec les propriétaires de chien. Les données étaient celles de la base de données du système de surveillance épidémiologique. Leur utilisation ne permet pas de révéler l'identité des propriétaires de chiens ou de personnes mordues. Nous avons obtenu l'autorisation de la DNSV et du LCV pour exploiter cette base de données.

\section{Résultats}

La figure 1 montre le diagramme de flux des données. La base comportait un total de 511 données dont 204 cas de rage avec données complètes, d'où une incidence cumulée, de 2009 à 2018 , de 39,92\%.

Le tableau I donne la répartition des Chiens en fonction des caractéristiques de l'animal selon l'âge, le sexe, I'habitat. La moyenne d'âge des chiens mordeurs, sur les 204 échantillons révélés positifs à l'immunofluorescence directe, est de 2,78 ans $\pm 2,17$. Le sexe mâle était le plus dominant avec $81,86 \%$. Bamako a enregistré la plus grande fréquence avec $63,24 \%$. Le tableau montre qu'il y a beaucoup plus cas de morsure en milieu urbain $(72,55 \%)$ qu'en milieu rural $(27,47 \%)$.

Dans notre échantillon, l'agressivité était le signe clinique le plus fréquent avec 74,02\% (tableau II). II était suivi par la fureur avec $57,35 \%$. La moitié des chiens $(50,98 \%)$ avait présenté une modification de voix.

Parmi les chiens vaccinés, $88,89 \%$ étaient du milieu urbain contre $11,11 \%$ pour le milieu rural (tableau III). La différence n'était pas significative. 
La fréquence de la morsure n'était pas liée à la saison (tableau IV). Les cas de morsure étaient légèrement plus fréquents en saison sèche $(95,20 \%)$ qu'en saison pluvieuse $(94,94 \%)$.

L'analyse du tableau $\mathrm{V}$ montre que les abatages et les cas de mort étaient plus élevés à Bamako que dans les autres régions. Le District de Bamako était suivi par la région de Koulikoro où nous avons enregistré $77,50 \%$ de chiens abattus et $22,50 \%$ cas de chiens morts. Les méthodes d'euthanasie et d'étouffement ne sont utilisées que dans le District de Bamako.

\section{Discussion}

Limite de l'étude: Dans l'échantillon, 37 données ont été supprimées fautes d'incomplétude. Cela sous estimerait l'incidence calculée et créé un biais de sélection.

L'incidence de la rage était de $39,92 \%$. Elle a été sousestimée du fait que la base comportait des données incomplètes chez 37 cas. Ce qui pose le problème d'insuffisance de la qualité des données de ladite base. Les analyses de la base du Laboratoire Central Vétérinaire (LCV) réalisées entre 2000 et 2003 et entre 2006 et 2013 retrouvent des fréquences respectives de $98,3 \% \quad(119 / 121)$ et $92,9 \%(435 / 468) \quad(10,11)$. En République Centre Africaine, l'analyse de la base de données de la rage de 2012, avait identifié 69 cas confirmé sur les 82 échantillons de cas suspect de rage animale d'où une fréquence de $84,1 \%$ (12).

Dans notre étude, les chiens mâles étaient les plus atteints de rage. Cela pourrait résulter du fait du regroupement des mâles autour des femelles pendant les périodes de rut des chiennes (13). Lors de ce regroupement, les mâles bagarrent entre eux et se mordent les uns des autres.

Presque trois quarts des chiens avaient présenté un signe d'agressivité, en faveur d'une rage furieuse et la fréquence du signe de fureur corrobore ce constat. La modification de voix qui fait partie des signes d'appel de l'imminence de la mort a été retrouvée chez la moitié des chiens. L'agressivité et l'agitation ont été rapportées par Dao $S$ et al. avant la mort des chiens enragés (10).

La prévalence de la vaccination chez les chiens de notre étude était très faible, seulement de $4,41 \%$. Cette fréquence était aussi beaucoup plus représentée en milieu urbain qu'en milieu rural. Ces résultats seraient en faveur de l'insuffisance de perception de l'importance de la vaccination des chiens par nos populations aussi bien rurales qu'urbaines. En milieu urbain le léger mieux s'expliquerait par le fait que la plus part des chiens sont enfermés ou avec un meilleur suivi par leurs propriétaires, contrairement au milieu rural où les chiens sont beaucoup plus errants. Dans l'étude de Traoré A et al., $45 \%(n=306)$ des chiens avaient été reportés comme vaccinés dont seulement $19,3 \%$ à jour à travers l'examen de la carte (11).

Les chiens étaient beaucoup plus atteints de rage durant la saison sèche soit un total de 61,27\%. Cela serait lié à période de rentrer en chaleur des chiennes. Cependant nous n'avons pas trouvé de différence de fréquence entre les deux saisons.

Les abatages étaient beaucoup plus fréquents à Bamako, probablement du fait que les services techniques vétérinaires y sont beaucoup plus sollicités. Nous avons aussi remarqué que les techniques de l'étouffement et de l'euthanasie n'étaient pratiquées qu'à Bamako. La pratique de l'étouffement serait faite par le propriétaire du chien. Cependant, celle de l'euthanasie serait liée à la présence des écoles de santé vétérinaire, la possibilité de choix de la technique mise à mort par le propriétaire du chien et surtout la présence des associations de défense des droits des animaux. Ces associations défendent la mise à mort en douce pour éviter la souffrance de l'animal.

\section{Conclusion}

L'incidence de la rage était élevée La plupart étaient des males. Le risque de faire la rage était plus élevé durant la saison sèche. Les signes cliniques dominant étaient l'agressivité, suivie de la fureur et de la modification de voix. Cette étude suggère le remplissage complet de la base de données et la sensibilisation des populations sur l'importance de la vaccination des chiens.

\section{Références}

1. OIE. Code sanitaire pour les animaux terrestres. Chapitre 8.14 : Infection par le virus de la rage [Internet]. OIE; 2019 [cité 10 juill 2019]. Disponible sur: https://www.oie.int/fileadmin/Home/fr/Health_standards/t ahc/current/chapitre_rabies.pdf

2. OIE. Portail Rage: OIE - World Organisation for Animal Health [Internet]. OIE; [cité 28 mai 2019].

Disponible sur: https://www.oie.int/fr/sante-animale-dansle-monde/portail-rage/

3. World Health Organisation. WHO Expert

Consultation on Rabies, Second report, 18 au 20

Septembre 2012 [Internet]. Genève, Suisse: WHO; 2012

sept [cité 13 juin 2019] p. 150. Disponible sur:

https://apps.who.int/iris/bitstream/handle/10665/85346/97

89241209823_eng.pdf;jsessionid=BF5DB1D18922D7D9 OA3571B9E4EB0317? sequence $=1$

4. Evelyne $P$, Florence $C$. Epidémiologie de la rage animale-V2-18. OIE; 2004.

5. Cliquet F, Picard-Meyer E. Rabies and rabiesrelated viruses: a modern perspective on an ancient disease. Rev Sci Tech Int Off Epizoot. août 2004;23(2):625-42.

6. Organization WH. Protocole d'évaluation des systèmes nationaux de surveillance et de riposte concernant les maladies transmissibles : directives pour les équipes d' évaluation. 2002 [cité 28 déc 2019]; Disponible sur: https://apps.who.int/iris/handle/10665/67234 
7. DNSV. Textes Législatifs et Réglementaires du domaine de Santé Publique Vétérinaire. DNSV; 2006.

8. Cameron A. Manuel de Surveillance des Principales Maladies Animales. Nairobi, Kénya : UA-Bira [Internet]. Union Africaine, Bureau Interafricaine des Ressources Animales; 2012 [cité 20 juin 2019]. Disponible sur: https://www.google.com/search 9. DNPIA. Rapport annuel. Bamako, Mali: DNPIA; 2018.

10. Dao S, Abdillahi A, Bougoudogo F, Toure K, Simbe $C$. Epidemiological aspects of human and animal rabies in the urban area of Bamako, Mali. Bull Société Pathol Exot 1990. 1 août 2006;99:183-6.

11. Traoré A, Picard-Meyer E, Mauti S, Biarnais M, Balmer O, Samaké K, et al. Molecular Characterization of Canine Rabies Virus, Mali, 2006-2013. Emerg Infect Dis. 1 mai 2016;22.

12. Tricou V, Bouscaillou J, Mebourou EK, Koyanongo FD, Nakouné E, Kazanji M. Surveillance of Canine Rabies in the Central African Republic: Impact on Human Health and Molecular Epidemiology. PLoS Negl Trop Dis. 9 févr 2016;10(2):e0004433.

13. AKAKPO A, ALAMBEDJI R. Aspects épidémiologiques et cliniques de la rage animale au Sénégal. 2001.

Remerciements : Gouvernement malien (Ministère de la Santé et des Affaires Sociales, Ministère de l'Elevage et de la Pêche, Ministère de l'Environnement); CDC/AFENET pour le financement de cette activité ; USTTB, DER de Santé Publique ; Formateurs et mentors pour leur accompagnement.

Liste des figures et tableaux :

Tableau V: répartition des Chiens en fonction des caractéristiques sociodémographiques au Mali de 2009 à 2018

\begin{tabular}{|c|c|c|}
\hline Caractéristiques & $\mathrm{n}$ & $\%$ \\
\hline Age & 204 & \\
\hline Moyenne & 2,78 & \\
\hline Mode & 1,00 & \\
\hline
\end{tabular}

\begin{tabular}{clll}
\hline & Médiane & 2,00 & \\
Ecart type & 2,17 & \\
Eexe & Etendue & $0,50-12,00$ & \\
& Mâle & 204 & 100,00 \\
Femelle & 167 & 81,86 \\
Région & 37 & 18,14 \\
Bamako & 204 & 100,00 \\
Gao & 129 & 63,24 \\
Kayes & 1 & 0,49 \\
Koulikoro & 2 & 0,98 \\
Mopti & 40 & 19,61 \\
Ségou & 15 & 0,98 \\
Sikasso & 15 & 7,35 \\
Milieu & 204 & 7,35 \\
Urbain & 148 & 100,00 \\
Rural & 56 & 72,55 \\
Vaccination & 204 & 27,45 \\
Oui & 9 & 100,00 \\
Non & 195 & 4,41 \\
Saison de morsure & 204 & 95,59 \\
Sèche & 125 & 100,00 \\
Pluvieuse & 79 & 38,27 \\
\hline
\end{tabular}

Tableau VI: Répartition des chiens vaccinés en fonction des signes cliniques au Mali de 2009 à 2018 (N=204)

\begin{tabular}{lll}
\hline Signe & $\mathbf{n}$ & $\mathbf{\%}$ \\
\hline Agressivité & 151 & 74,02 \\
Hypersalivation & 46 & 22,55 \\
Fureur & 117 & 57,35 \\
Excitation génésique & 46 & 22,55 \\
Modification de la voix & 104 & 50,98 \\
\hline
\end{tabular}

Tableau VII : Répartition des chiens vaccinés en fonction du milieu au Mali de 2009 à 2018

\begin{tabular}{lllll}
\hline \multicolumn{2}{l}{ Vaccination/ Milieu } & Urbain & Rural & $\mathbf{p}$ \\
\hline \multirow{2}{*}{ Vacciné } & Oui & $8(88,89)$ & $1(11,11)$ & 0,44 \\
& Non & $140(71,79)$ & $55(28,21)$ & \\
\hline
\end{tabular}

Tableau VIII : Répartition des cas de morsure en fonction des saisons

\begin{tabular}{llll}
\hline Saison/Morsure & Oui $\mathbf{n}(\%)$ & Non $\mathbf{n}(\%)$ & $\mathbf{p}$ \\
\hline Sèche & $119(95,20)$ & $6(4,80)$ & 0,99 \\
Pluvieuse & $75(94,94)$ & $4(5,06)$ & \\
\hline
\end{tabular}




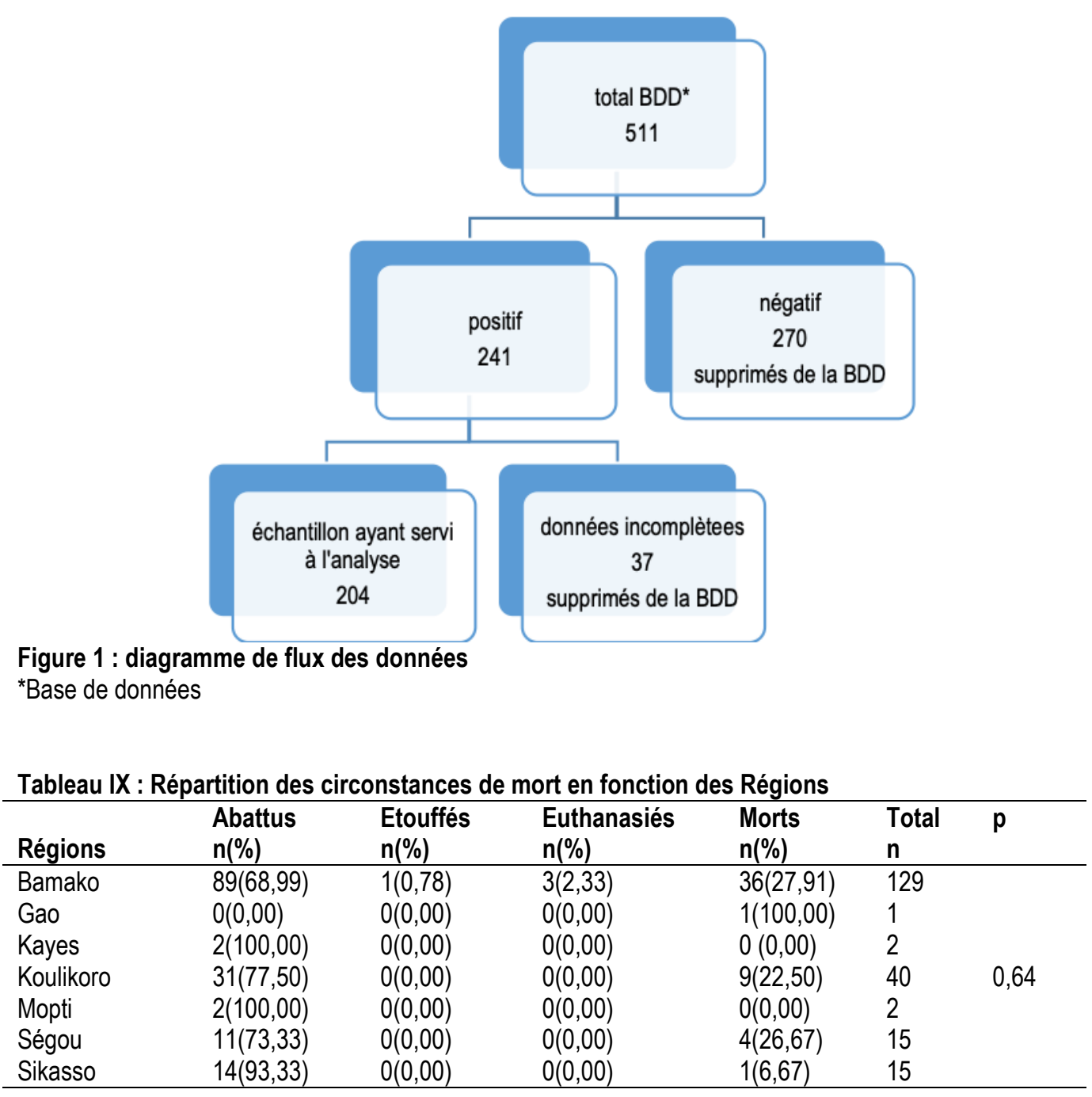




\section{INSTRUCTIONS AUX AUTEURS}

La revue Mali Santé Publique (MSP) publie des éditoriaux, des articles originaux, des revues de la littérature sous forme de mise au point, des pratiques, des opinions et débats sous forme d'analyse des politiques de santé, des programmes de santé ou interventions.

\section{CONDITIONS GENERALES DE PUBLICATIONS}

Les articles n'engagent que la seule responsabilité de leurs auteurs. Les manuscrits des articles ne doivent avoir fait l'objet d'aucune publication antérieure, ni être soumis pour publication à une autre revue scientifique. Les textes de bonne qualité saisis en interligne 1.5, de police 12 en Times New Roman, ainsi que les photos, figures et tableaux, doivent être soumis en ligne sur le site de la revue ou à l'adresse mali.sante.publique@gmail.com, akory.agiknane@gmail.com ou en version papier. L'article doit rester anonyme. Dans un fichier séparé, seront mentionnés le nom des auteurs et l'adresse complète du correspondant afin de lui adresser une correspondance après l'avis des lecteurs. En cas de soumission en version papier, 3 exemplaires seront déposés au secrétariat du DER de Santé Publique à la Faculté de Médecine au Point G. Deux des exemplaires ne comporteront ni noms ni adresses des auteurs.

Les auteurs doivent préciser dans quelle rubrique ils soumettent leur article. Tous les articles sont adressés pour avis de façon anonyme à deux lecteurs. Un courrier accompagnant les avis de lecture sera adressé à l'auteur correspondant dans un délai maximum de 30 jours après la réception de l'article. Ce dernier doit renvoyer à la rédaction son texte corrigé suivant les recommandations des lecteurs dans un délai d'un mois. Si le texte est définitivement accepté par la rédaction, celle-ci procédera aux modifications minimes (orthographe, style, mise en page).

\section{PRESENTATION DES TEXTES}

La première page de chaque manuscrit doit, quelque soit la rubrique proposée, comporter : le titre de l'article (maximum 20 mots, en Français et en Anglais), les initiales des prénom et les noms des auteurs, la dénomination complète de l'institution dans laquelle le travail a été effectué, les titres et affiliations de chaque auteur, l'adresse complète avec numéros de téléphone et de fax et adresse email. Cette première page doit être soumise sous la forme d'un fichier séparé.

Les marges sont de 2,5cm de chaque côté. La numérotation des pages est en bas à droite.

Les éditoriaux sont généralement sollicités par la rédaction. Les manuscrits n'excèdent pas 4 pages (références non comprises mais limités à 10).

Les articles originaux ne doivent pas dépasser 12 pages (références non comprises, de 10 à au moins). Ils nécessitent une introduction précisant clairement le but de l'étude, un chapitre matériel et méthodes suivi des résultats, une discussion et une conclusion avec recommandations.

La revue de la littérature ou Mise au point, le manuscrit ne doit pas dépasser 16 pages, les références non incluses peuvent être exhaustives.

Les opinions ou débats sont encouragés, le manuscrit est limité à 4 pages, les références à 5 .

Dans tous les cas, les abréviations doivent êtres évitées, si non indiquées à leur premier emploi, après l'expression ou le mot qu'elles abrègent. 


\section{RESUME}

Les articles originaux, les mises au point doivent s'accompagner d'un résumé en Français et en Anglais suivi de 3 à 5 mots clés. Adressé en 3 exemplaires, le résumé doit figurer sur une page à part du reste du manuscrit. D'une longueur maximale de 250 mots, il ne doit comporter ni abréviations, ni références.

Pour les articles originaux, il doit préciser les objectifs, la méthodologie, les résultats et la conclusion. Tout manuscrit non accompagné de résumé en Français et en Anglais n'est pas recevable par la rédaction.

\section{LES REFERENCES}

Les références sont numérotées selon l'ordre de leur appel dans le texte en conformité aux normes uniformisées pour la préparation des manuscrits soumis aux journaux biomédicaux, selon le style de Vancouver. Les logiciels de référencement, tel que Zotero, sont privilégiés. Elles doivent indiquer les noms de tous les auteurs jusqu'à 6 , au-delà ajouter « et al. ». Seuls les articles déjà publiés ou sous presse peuvent être mentionnés. La liste des journaux et de leurs abréviations peut être consultée en ligne, sur le serveur PubMed, à l'adresse suivante : http://www.ncbi.nlm.nih.gov/nlmcatalog/journals. Dans le texte et les tableaux, les références sont numérotées en chiffres arabes entre crochets/parenthèses dans l'ordre d'apparition. Quant plus de deux se suivent, indiquez le premier et le dernier chiffre en les séparant par un tiret [7-11]. Les communications personnelles ou les données non publiées ne figureront pas dans la liste des références, mais peuvent être mentionnées entre parenthèse dans le texte.

Les références sont dactylographiées en interligne et son présentées selon les indications suivantes :

\section{Articles de périodiques :}

HOGSTEDT C, LUNDBERG I. Epidemiology of occupational neurobehavioural hazads: Methodological experiences from organic solvent research. Rev Epidemiol Sante Publique. 1992; 40: 7-16.

\section{Ouvrages}

FLEISS JL. Statistical methods for rates and proportions. $2^{\text {nd }}$ ed. New York: John Wiley and Sons; 1981.

AG IKNANE A, CAMARA I, KADJOKE M, CAMARA M. La santé communautaire au Mali: Aspects juridiques, réglementaires et économie politique. Bamako : Edition Jamana; 2000.

\section{Chapitre d'ouvrages}

Coulibaly So, Diarra K. Analyse des comptes nationaux de la santé. Dans : Se soigner au Mali, une contribution des sciences sociales. Paris : Karthala - ORSTOM ; 1993. :p. 231-246.

DIAKITE BD, DIARRA T, TRAORE S. Recours aux soins et consommation médicale dans le quartier de Bankoni. Dans : Se soigner au Mali, une contribution des sciences sociales. Paris:Karthala , ORSTOM,;1993 : (155-175). 


\section{DIRECTIVES DE LA REVUE Mali Santé Publique (MSP) \\ POUR L'ÉVALUATION D'UN MANUSCRIT}

Dossier $\mathrm{N}^{\circ}$

Titre :

Date de retour :

Nombre de mots :

N.B. : le nombre maximum de mots pour un texte à publier dans la Revue Mali Santé Publique (sans compter le résumé, les tableaux, les figures et les références bibliographiques) est de 2000 mots

\section{Renseignements généraux :}

Les réponses que vous ferez aux questions suivantes à propos du manuscrit ci-joint vous aideront à déterminer s'il y a lieu de le publier dans la revue Mali santé Publique. Après avoir lu le manuscrit, prière de répondre aux questions en cochant votre réponse. Si vous désirez nuancer votre réponse, prière de le faire à la section des observations particulières, à la fin du questionnaire. Si vous répondez « Non », « incertain » ou « Amélioration requise», une explication détaillée s'impose pour aider les rédacteurs et les auteurs.

\section{RESUME :}

La matière abordée est-elle pertinente pour la RMSP?

1. le problème est-t-il clairement énoncé?
$\square$ Bon
$\square$ Amélioration requise
$\square$ Non

2. La méthodologie de l'étude est-elle clairement exposée?
$\square$ Bon
$\square$ Amélioration requise
$\square$ Non

3. Des résultats importants sont-ils clairement exposés?
$\square$ Bon
$\square$ Amélioration requise
$\square$ Non

4. Les principales conclusions et recommandations sont-elles clairement exposées?
$\square$ Bon
$\square$ Amélioration requise
$\square$ Non

\section{INTRODUCTION}

5. Le problème est-il correctement énoncé?

6. Le problème énoncé est-il pertinent?
$\square$ Bon
$\square$ Amélioration requise
$\square$ Non

$\square$ Bon $\square$ Amélioration requise $\quad \square$ Non

7. Une documentation pertinente est-elle succinctement analysée et citée?
$\square$ Bon
$\square$ Amélioration requise
$\square$ Non

8. La question de recherche ou l'hypothèse est-t-elle clairement énoncée?
$\square$ Bon
$\square$ Amélioration requise
$\square$ Non

\section{METHODES}

9. Les méthodes telles qu'exposées permettraient-elles à d'autres chercheurs de reprendre l'étude?
$\square$ Bon
$\square$ Amélioration requise
$\square$ Non

10. Les méthodes utilisées conviennent-elles à la question de recherche?
$\square$ Bon
$\square$ Amélioration requise
$\square$ Non

11. Les méthodes statistiques utilisées conviennent-elles à la question de recherche?
$\square$ Bon
$\square$ Amélioration requise
$\square$ Non 


\section{RESULTATS}

12. Les résultats sont-ils clairement présentés?
$\square$ Bon
$\square$ Amélioration requise
$\square$ Non

13. Les résultats sont-ils pertinents par rapport au problème de recherche?

14. Les tableaux et figures sont-ils adéquats et clairs?
$\square$ Bon
$\square$ Amélioration requise
$\square$ Non

$\square$ Bon $\square$ Amélioration requise $\square$ Non

15. Les chiffres sont-ils crédibles (c'est-à-dire vraisemblables?)
$\square$ Bon
$\square$ Amélioration requise
$\square$ Non

\section{DISCUSSIONS}

16. L'interprétation et les conclusions s'appuient-elles sur les résultats?
$\square$ Bon
$\square$ Amélioration requise
$\square$ Non

17. Les résultats obtenus sont-ils comparés à ceux d'autres études antérieures?
$\square$ Bon
$\square$ Amélioration requise
$\square$ Non

18. Les limites de l'étude (s'il y en a) et de ses résultats sont-ils expliqués?
$\square$ Bon
$\square$ Amélioration requise
$\square$ Non

\section{REFERENCES}

19. Les références sont-elles pertinentes?
$\square$ Bon
$\square$ Amélioration requise
$\square$ Non

\section{CONSIDERATIONS GENERALES}

20. S'agit-il d'une priorité de recherche nationale?
$\square$ Bon
$\square$ Amélioration requise
$\square$ Non

21. Le manuscrit est-il bien présenté (y compris le style rédactionnel)?
$\square$ Bon
$\square$ Amélioration requise
$\square$ Non

DECISION FINALE (Veuillez cocher l'une des réponses suivantes.)

Le publier tel quel, en priorité.

Le publier tel quel, sans priorité.

Le retourner à l'auteur pour être retouché. (Précisez quelles seraient les retouches à y apporter.)

Conseiller à l'auteur de le reprendre entièrement en lui proposant un appui technique afin de l'amener à produire un article convenable.

\section{OBSERVATIONS}

Les observations que vous inscrirez sur cette fiche et tout autre commentaire que vous jugez pertinent pour l'article à évaluer nous aideront à prendre une décision concernant la publication du manuscrit. Si vous avez d'autres observations, nous vous encourageons à les écrire sur une ou plusieurs pages supplémentaires.

Ne vous préoccupez pas de corriger les coquilles ou les fautes de grammaire, car elles seront relevées par le comité de rédaction. Merci de votre aide. 


\section{SOCiete MAlienne de SANte Publique}

La Revue Mali Santé Publique (RMSP) est éditée par la Société Malienne de Santé Publique (SOMASAP).

Parution Semestrielle

Adresse Site web : http://revues.ml/index.php/msp/about

Email : mali.sante.publique@gmail.com ou akory.agiknane@gmail.com

Tel : (223) $66760075 / 76486975 / 66759051$

Orange Money : 76412068

Imprimerie: SODOFI - Sarl 34, Rue 373 (Fankelé Diarra), Bamako-coura BP : 1866 Tel : 20226140 Fax : 20238558 E-mail : $\underline{\text { sodifi@cefib.com }}$

$N^{\circ}$ ISSN - 1987-0728

Juin 2019 